\title{
Requirements for self-magnetically insulated transmission lines
}

\author{
J. Pace VanDevender, ${ }^{1,2, *}$ Timothy D. Pointon, ${ }^{1}$ David B. Seidel, ${ }^{1}$ Kenneth W. Struve, ${ }^{1}$ \\ Christopher Jennings, ${ }^{1}$ Bryan V. Oliver, ${ }^{1}$ and Larry X. Schneider ${ }^{1}$ \\ ${ }^{1}$ Sandia National Laboratories, P.O. Box 5800, Albuquerque, New Mexico 87185-1186, USA \\ ${ }^{2}$ VanDevender Enterprises, 7604 Lamplighter Lane NE, Albuquerque, New Mexico 87109, USA \\ (Received 23 November 2013; revised manuscript received 31 December 2014; published 2 March 2015) \\ Self-magnetically insulated transmission lines (MITLs) connect pulsed-power drivers with a load. \\ Although the technology was originally developed in the 1970s and is widely used today in super power \\ generators, failure of the technology is the principal limitation on the power that can be delivered to an \\ experiment. We address issues that are often overlooked, rejected after inadequate simulations, or covered \\ by overly conservative assumptions: (i) electron retrapping in coupling MITLs to loads, (ii) the \\ applicability of collisionless versus collisional electron flow, (iii) power transport efficiency as a function \\ of the geometry at the beginning of the MITL, (iv) gap closure and when gap closure can be neglected, and \\ (v) the role of negative ions in causing anode plasmas and enhancing current losses. We suggest a practical \\ set of conservative design requirements for self-magnetically insulated electron flow based on the results \\ discussed in this paper and on previously published results. The requirements are not necessarily severe \\ constraints in all MITL applications; however, each of the 18 suggested requirements should be examined \\ in the design of a MITL and in the investigation of excessive losses.
}

DOI: 10.1103/PhysRevSTAB.18.030401

PACS numbers: 52.75.-d, 52.27.Jt, 52.58.Lq

\section{INTRODUCTION}

Experiments on multimodule, pulsed-power accelerators are providing new insights into high-energy-density physics [1], isentropic compression [2], shock physics [3], inertial confinement fusion [4], and radiation effects simulation [5]. These experiments require tens of millions of amperes to be delivered to a common load through load-dominated, selfmagnetically insulated transmission lines (MITL) and selfmagnetically insulated inductive elements called convolutes [6-9]. MITLs have simple geometries-coaxial, parallel plate, triplate, and biconic-with impedances that are constant or vary little with distance into the MITL. The term "convolute" is derived from the verb convolute and means any complex geometry of interwoven anodes and cathodes connecting two simpler transmission lines. They are useful for minimizing inductance when the anodecathode gap of a matched impedance transmission line would either break down electrically or is impractical mechanically. The posthole convolute (PHC) [6-9] is shown in Fig. 1(a) and is the most developed convolute geometry. It worked well for a generation of pulsed-power machines. However, poorly understood current losses in the PHC severely limit the load current in the highest power accelerators [10-12] and present an extremely challenging power

*pace@vandevender.com

Published by the American Physical Society under the terms of the Creative Commons Attribution 3.0 License. Further distribution of this work must maintain attribution to the author(s) and the published article's title, journal citation, and DOI. flow problem. The complexity of the PHC makes a detailed study of its performance very difficult. The simpler convolute shown in Fig. 1(b) allowed us to study the electron flow and efficiency of a convolute with a relevant (spatially varying impedance causing complex electron flow in three dimensions) but more understandable geometry.

In addition, the recent development of linear transformer drivers (LTDs) [13] has renewed interest in the transport of energy in long self-limited MITLs [14].

Advances in the theory and practice of MITL power flow since the 1970s, the losses in PHCs, and the renewed interest in multimodule, self-magnetically insulated LTD-based accelerators driving a common inductive load motivated reexamination of the requirements for efficient MITLs. The results of the magnetically insulated transmission experiment (MITE) [15] from the 1970s provided relevant data on the magnitude and distribution of losses for various MITL configurations and load impedances and are briefly summarized in Sec. II. In Sec. III, those results are interpreted in light of the subsequent theoretical advances on magnetic insulation. Simulations of the MITE results with a modified Screamer circuit code test the requirements for efficient energy transport in MITLs. MITL design requirements, the principal purpose of this paper, are presented in Sec. IV.

New analyses of previously published work contributed to the requirements and illuminated issues that are often covered by overly conservative assumptions: "All the electron flow is lost before the load" is inconsistent with the results in Secs. III A and III B, "collisional electron flow is a worst case" is true but the data in Sec. III B support collisionless flow, "the geometry at the beginning of a 
(a)
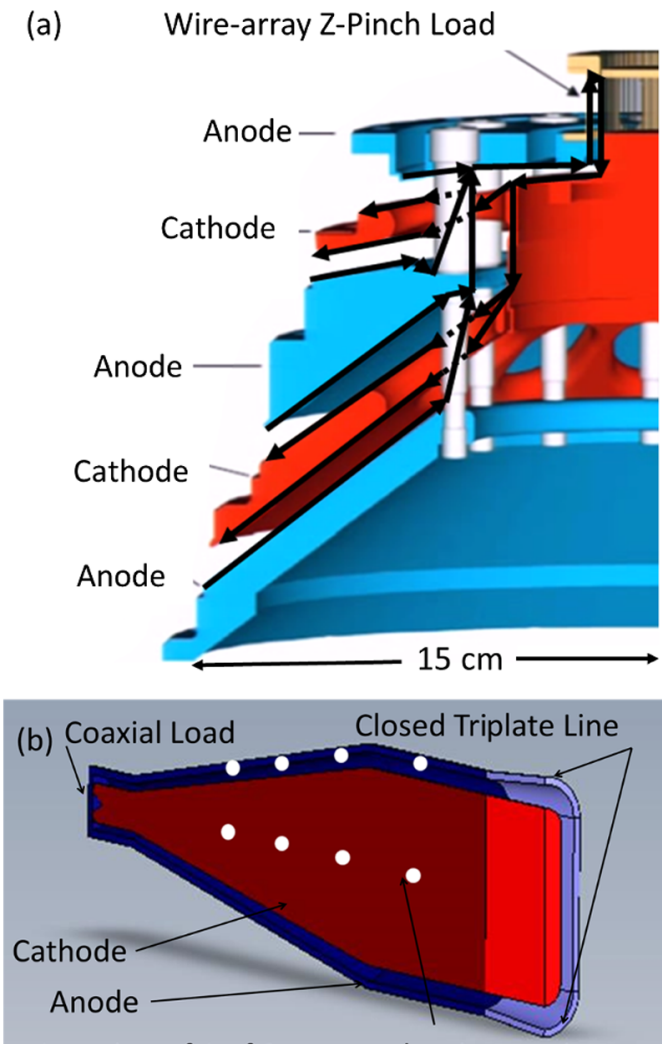

Location of 1 of 8 current density monitors

FIG. 1. (a) Quarter cross-sectioned view of a double PHC with 12 -fold azimuthal symmetry. The currents from double biconic MITLs are combined to power the common $z$-pinch load on the $Z$ Machine at Sandia National Labs. The three-dimensional complexity is evident from the arrows, which show the current path from the anodes to the cathodes; dotted lines indicate flow through the cathode behind the anode post. (b) Cross-sectioned view of the simpler, MITE tapered convolute, as seen in a cutaway view, through the vertical plane of symmetry.

MITL is not important because the simulations show no effect on the electron flow" is inconsistent with the data in Sec. III C, and "anode-cathode gaps close at $\sim 2.5 \mathrm{~cm} / \mu \mathrm{s}$ " is inconsistent with data discussed in Sec. IV, point 3. In addition, the potential for negative ions to form anode plasmas and enhance positive ion current losses in PHCs is proposed in Sec. IV, point 18.

We offer the 18 requirements in Sec. IV as a guide for a conservative MITL design and as an aide in researching unexpected losses. A particular MITL design may function satisfactorily without meeting one or more of these requirements. Indeed, the MITL-PHC design on the $Z$ Machine does not satisfy at least three of the requirements and functioned satisfactorily until the pulsed power was increased substantially in the $Z$ Refurbishment project.

\section{UPDATED MITE RESULTS}

The magnetically insulated transmission experiment (MITE) culminated in the first efficient self-limited
MITL [15] and formed the basis for the Particle Beam Fusion Accelerator I (PBFA I). Although PBFA I was designed to have 36 independent electron beams, the shift from electron beam fusion to ion beam fusion shortly before PBFA I was completed required the combination of the 36 MITLs into a common disk feed [16]. The combination was reasonably efficient for low-impedance loads, but it failed to efficiently power the high-impedance ion-diode loads [17]. Therefore, a load-dominated MITL with a PHC was adopted for PBFA II and subsequent accelerators. At that time, the MITE results were incorrectly assumed to be irrelevant to load-dominated MITLs.

\section{A. Tapered convolute efficiency}

Although MITE had a 6-m-long self-limited MITL, the MITE output convolute experiment [16] was a short, load-dominated MITL shown in Fig. 1(b). Anode and cathode currents were measured just before and after the tapered convolute. The measurements let us test the assumption $[7,11]$ that all of the electron flow going into a convolute is lost before it reaches the load. We find that substantial retrapping of electrons occurs.

In Fig. 1(b), self-magnetically insulated power flow is incident from the right, where the vertical triplate geometry is visible, and converges through the tapered triplate convolute, to the coaxial MITL, and then to the pinched electron beam diode on the left. The red cathode in Fig. 1(b) is surrounded by the blue anode. The self-magnetic field lines enclose all the current in the cathode. The white circles show the locations of monitors for the electron loss.

The anode-cathode gap is $1.0 \mathrm{~cm}$ on the sides and $2.0 \mathrm{~cm}$ on the top and bottom in the 6-m-long, closed triplate MITL, of which $30 \mathrm{~cm}$ is shown in Fig. 1(b) as the power feed into the tapered convolute. The height of the planar portion of the center MITL electrode (cathode) is $25.4 \mathrm{~cm}$. Its radius is $2.54 \mathrm{~cm}$ on the top and bottom and is maintained in the 36-cm-long, linearly tapered convolute. The top and bottom anode-cathode gaps taper from $2.0 \mathrm{~cm}$ at the beginning of the convolute to $1.0 \mathrm{~cm}$ at the output. The anode-cathode gap is $1 \mathrm{~cm}$ on the sides. The resulting profile of the vacuum wave impedance from the output of the convolute at $z=0.1 \mathrm{~m}$ from the load to the input of the convolute at $z=0.46 \mathrm{~m}$ is shown in Fig. 2.

The tapered convolute terminates in a 10-cm-long, coaxial MITL with an inner radius of $2.54 \mathrm{~cm}$ and a $1.0 \mathrm{~cm}$ gap. The geometrical vacuum wave impedance is $20 \Omega$ in the coaxial region, but the azimuthally asymmetric power feed increases the effective impedance at $10 \mathrm{~cm}$ from the load to approximately $24 \pm 3 \Omega$. The load is a taperedcathode, electron beam diode with a conical hole from $r=0$ to $1.27 \mathrm{~cm}$ to avoid impedance reduction by anodeplasma motion. The load impedance was adjusted by changing the taper of the cathode and the anode-cathode gap and was in general agreement with Creedon's theory [18]. 


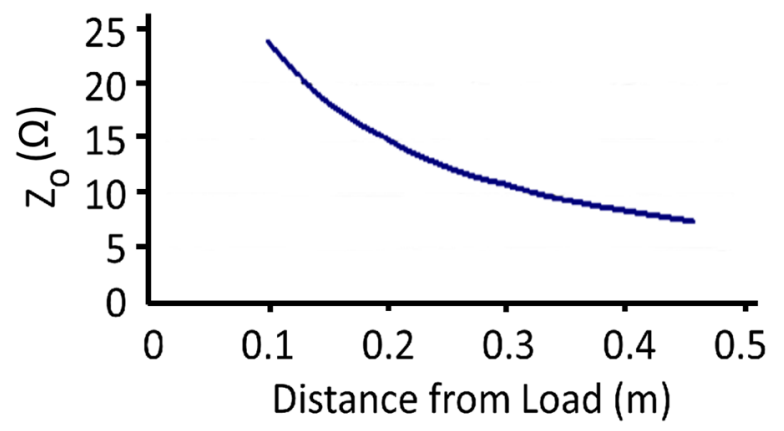

FIG. 2. Approximate variation of the vacuum wave impedance with distance from the electron beam load in the MITE convolute.

The voltage and current at the input are approximately $1.8 \mathrm{MV}$ and $375 \mathrm{kA}$, respectively. Although the vacuum wave impedance $Z_{o}$ was $7.6 \Omega$, the MITL operated at $4.8 \Omega$ because of the electron current flowing in the anode-cathode gap. The power transport to the output convolute is quite efficient [15]. However, the current transport efficiency through the convolute [16] was $\sim 80 \%$ for matched load impedance $Z_{\mathrm{Load}}=4.8 \Omega$ or less and fell off as approximately $1 / Z_{\text {Load }}$ for $Z_{\text {Load }}>$ $4.8 \Omega$, which corresponds to $Z_{\mathrm{Load}} / Z_{\mathrm{o}_{-} \min }>0.63$ in Fig. 3. $Z_{\mathrm{O}_{-} \min }$ is the minimum vacuum wave impedance in the MITL.

The difference between the curve labeled MITE data and the curve labeled $Z_{\text {Flow }}$ model in Fig. 3 is the substantial retrapping of electron flow and contradicts the assumption of inefficient retrapping.

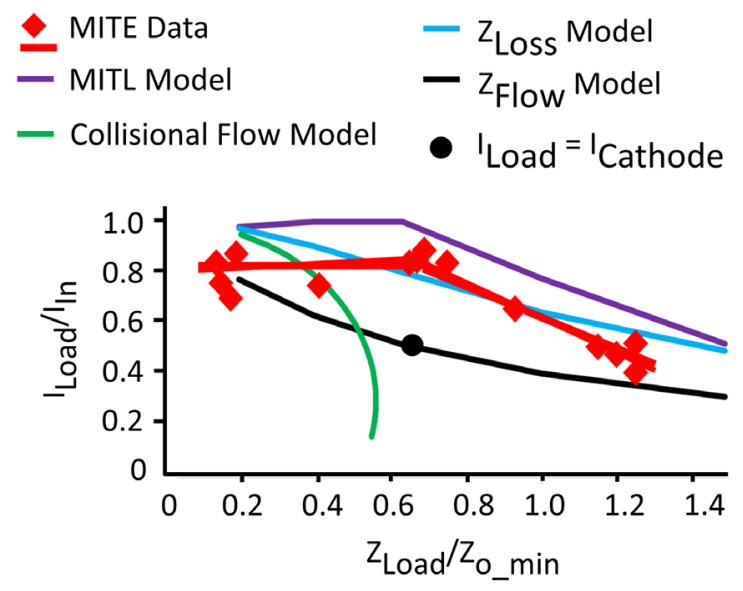

FIG. 3. The measured and simulated current transport efficiency $I_{\text {Load }} / I_{\text {In }}$ is shown as a function of the load impedance normalized by the minimum vacuum wave impedance $Z_{o}=$ $7.6 \Omega$ of the MITE MITL at the input to the convolute. The matched load is at $4.8 \Omega$ for $Z_{\mathrm{Load}} / Z_{o}=0.63$. The best fit to the data is shown as the bold red line. The black circle shows the expected value for the worst-case assumption that all the electron flow is lost before the load. Solutions for the collisional case are limited to lower impedance loads.

\section{B. Distribution of losses in MITE tapered convolute}

The spatial distribution of the time integrated loss in the convolute was computed from thermoluminescent dosimetry (TLD) measurements of the x-ray dose at eight locations on the taper, as shown in Fig. 1(b). All data were taken with matched load impedance. Electron energy spectroscopy on previous measurements in this configuration showed that the effective voltage and electron energy was reliably $1.8 \pm 0.2 \mathrm{MV}$. The time-averaged, losscurrent densities inferred from the TLD data are shown in Fig. 4.

The inferred loss current density is much greater on the top than it is on the sides. By symmetry, the loss current density on the bottom should be the same as on the top. Since the loss-current-density weighted area on the top and bottom is approximately $170 \mathrm{~cm}^{2}$ and is small compared to the corresponding $1300 \mathrm{~cm}^{2}$ area on the sides, the loss is dominated (approximately 85\% is to the sides) by the loss to the sides. Simulations with collisionless electron flow in this experiment are consistent with the loss current density, the input voltage and current, and the load current.

\section{MITL injector}

The MITE project investigated the effect of the shape of the transition from a low field region to the highly stressed MITL. Electron emission first begins in the most highly stressed gap of the MITL; however, once the upstream regions emit electrons, the electron flow into the MITL affects the emission from the rest of the MITL through the associated space charge and current. We call this transition the injector. The results from two of the four injector geometries investigated on the MITE experiment illustrate the unexpected importance of the injector.

In both cases, the height of the inner triplate transmission line was $25.4 \mathrm{~cm}$. As shown in Fig. 5(a), the first injector transitioned from a 2-cm anode-cathode gap to the $1-\mathrm{cm}$ anode-cathode gap of the uniform-gap MITL over a distance of only $5 \mathrm{~cm}$. It operated with a normalized flow impedance $Z_{\mathrm{Flow}} / Z_{o}=0.6$, showed significant losses at 53-121 cm into the MITL, and had energy transport efficiency of $60 \% \pm 4 \%$ to the end of the 6-m-long

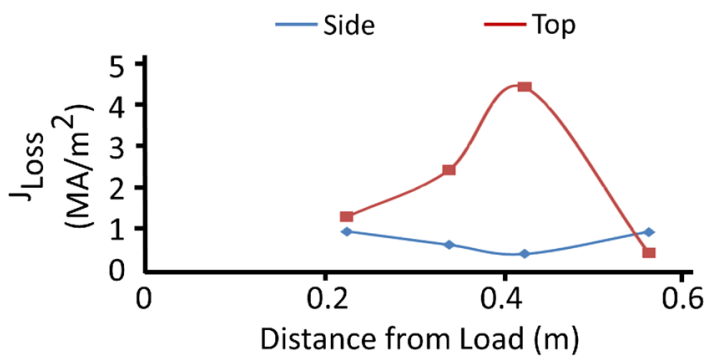

FIG. 4. Time-averaged, loss-current density as a function of distance from the load inferred from the TLD measurements for $1.8 \mathrm{MeV}$ electrons in a $35 \mathrm{~ns}$ pulse with a $4.8 \Omega$ load. 
(a)

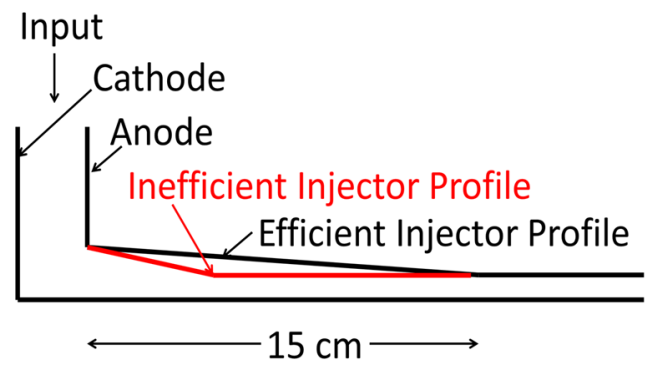

(b)

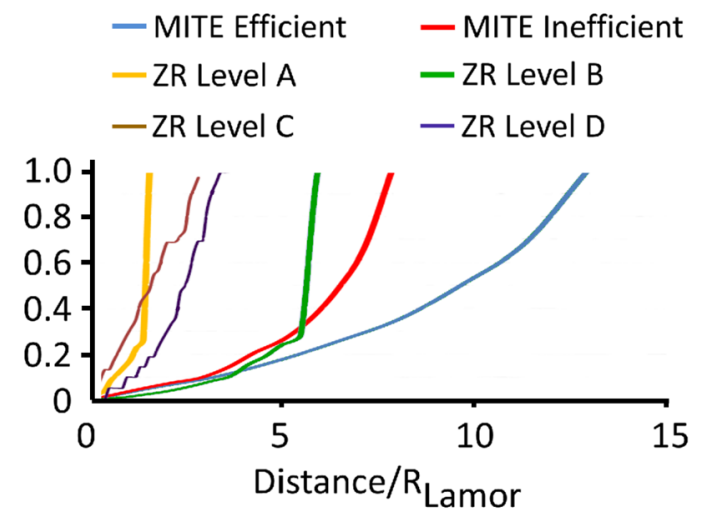

FIG. 5. (a) Cross section views of the inefficient and efficient injectors on MITE. (b) Normalized electron current versus normalized distance in the injector upstream from the uniformgap MITL in MITE for an efficient injector (gap is tapered from 2 to $1 \mathrm{~cm}$ over an axial distance of $15 \mathrm{~cm}$ ) and an inefficient injector (gap is tapered from 2 to $1 \mathrm{~cm}$ over an axial distance of $5 \mathrm{~cm}$ ). The similar plots for the four MITLs in the refurbished Z Machine at peak voltage on the vacuum insulator are shown for comparison with a modern, load-dominated MITL configuration.

MITL. The second injector geometry transitioned from a 2-cm anode-cathode gap to the $1-\mathrm{cm}$ anode-cathode gap of the uniform-gap MITL over a distance of $15 \mathrm{~cm}$, had a normalized flow impedance $Z_{\text {Flow }} / Z_{o}=0.71$, and had an energy transport efficiency of $>90 \% \pm 4 \%$. The only difference in the two sets of experiments was the abruptness of the injector geometry, which is characterized by the calculated electron current $I_{e}$, as a function of the distance from the uniform-gap MITL.

To facilitate comparison with other MITL geometries, the electron current is normalized to the electron current $I_{\mathrm{e}_{-} \text {final }}$ in the uniform-gap MITL. The distance scale is normalized to the Larmor radius $R_{\text {Larmor }}$ calculated at the anode midway through the injector. $R_{\text {Larmor }}=\beta \gamma m_{e} c /$ $(e B)$, for relativistic factors $\beta$ and $\gamma$, electron mass $m_{e}$, speed of light in vacuum $c$, electron charge $e$, and magnetic field $B$. The results are shown in Fig. 5(b).

The Larmor radii for MITE and $Z$ are typically 0.8 and $2.5 \mathrm{~cm}$, respectively. The blue line represents an efficient injector profile, and the red line represents the inefficient profile for a long, self-limited MITL. The corresponding profiles for the $Z$ Machine are discussed in Sec. IV.

\section{DISCUSSION OF MITE RESULTS}

The results from the MITE experiment predate many of the theoretical advances in self-magnetically insulated electron flow. We briefly summarize the key concepts from the subsequent theoretical development and then discuss the MITE results with respect to those developments and the associated assumptions.

In principle, positive ion emission from the MITL anode and negative ion emission from the MITL cathode can contribute to the loss current in MITLs. However, the loss current in MITLs is usually assumed to be electron current. The 90\% energy-transport efficiency in the 6-m-long MITL is consistent with only electron loss at the front of the pulse and places an upper bound on the positive and negative ion losses of $\ll 2 \%$ of monopolar space-charge-limited ion flow. Therefore, the assumption of electron-only loss is supported by the MITE data.

However, the current and power densities in MITE were much less than those in the PHC on multimodule generators like Z. In Sec. IV, points 9 and 18, the issue of positive and negative ion losses in highly stressed PHCs is reconsidered, after the case of electron-only losses has been discussed.

Mendel et al. [19] developed an important formalism for understanding collisionless electron flow in MITLs when the flow is held in equilibrium between the anode and cathode by the magnetic and electric energy densities (pressure and tension, respectively). The quantity $Z_{\text {Flow }}$ naturally arises in their formulation:

$$
Z_{\text {Flow }}=\frac{V}{I_{\text {upstream }}^{2}-I_{\text {downstream }}^{2}}=\frac{V}{I_{\text {anode }}^{2}-I_{\text {cathode }}^{2}},
$$

in which $V$ is the voltage between the anode and cathode and $I_{\text {upstream }}$ and $I_{\text {downstream }}$ are the currents measured upstream and downstream from the loss, respectively. $I_{\text {upstream }}$ equals the current in the cathode $I_{\text {cathode }}$ plus the current in electron flow $I_{e}$ and is the current measured at the anode $I_{\text {anode }}$. Since the entire electron current is usually assumed to be lost before it reaches the load, $I_{\text {downstream }}$ is usually assumed to equal $I_{\text {cathode, }}$, as shown in Eq. (1).

The fraction of the total current $I_{\text {anode }}$ that is in electron flow is readily obtained by rearranging Eq. (1) as

$$
\frac{I_{e}}{I_{\text {anode }}}=1-\sqrt{1-\frac{Z^{2}}{Z_{\text {Flow }}^{2}}},
$$

in which $Z=V / I_{\text {anode }}$.

Mendel et al. [20] derived an expression for $I_{e}$ for uniform electron density in the magnetically insulated flow. Ottinger and Schumer [21] have contributed a modification for nonuniform electron density. The resulting equation follows: 


$$
V=Z_{o}\left(I_{\text {anode }}^{2}-I_{\text {cathode }}^{2}\right)^{\frac{1}{2}}-g\left(\frac{m_{e} c^{2}}{2 e}\right)\left(\frac{I_{\text {anode }}^{2}-I_{\text {cathode }}^{2}}{I_{\text {cathode }}^{2}}\right),
$$

in which $g$ is the Ottinger and Schumer modification $g=0.99565-0.05332 \mathrm{~V} / V_{o}+0.0037\left(V / V_{o}\right)^{2}$, for $V_{o}=$ $1 \mathrm{MV}$, and is derived from computer simulations.

More recently, Stygar et al. [22] have extended the previous work, all of which assumed collisionless electron flow, to collisional magnetically insulated electron flow. They find that the relationship corresponding to Eq. (1) is not dependent on the value of the ratio $v_{\text {eff }} / \omega_{c}$ of collision frequency $v_{\text {eff }}$ to cyclotron frequency $\omega_{c}$ and develop a relationship between $V, I_{\text {anode }}$, and $I_{\text {cathode }}$ for collisional flow:

$$
V=\frac{I_{\text {anode }} Z_{o}}{2}\left(\left(\frac{\chi+1}{\chi-1}\right)^{\frac{1}{2}}-\frac{\ln \left[\chi+\left(\chi^{2}-1\right)^{\frac{1}{2}}\right]}{\chi(\chi-1)}\right),
$$

in which $\chi=I_{\text {anode }} / I_{\text {cathode }}$. The collisional electron flow fills the entire anode-cathode gap and, as Stygar et al. [22] note, represents a limiting worst case for the electron losses in a MITL.

Equations (3) and (4) represent equilibrium flow and give zero, one, or two values of $I_{\text {cathode }}$ for a given $V$ and $I_{\text {anode, }}$, as illustrated in Fig. 6. As Ottinger and Schumer [21] note, all of the solutions are possible, and the particular solution depends on how the flow is established. In their simulations, the electron flow was launched by an abrupt change in the electron emission in a section of a constantgeometry transmission line, which is very difficult to

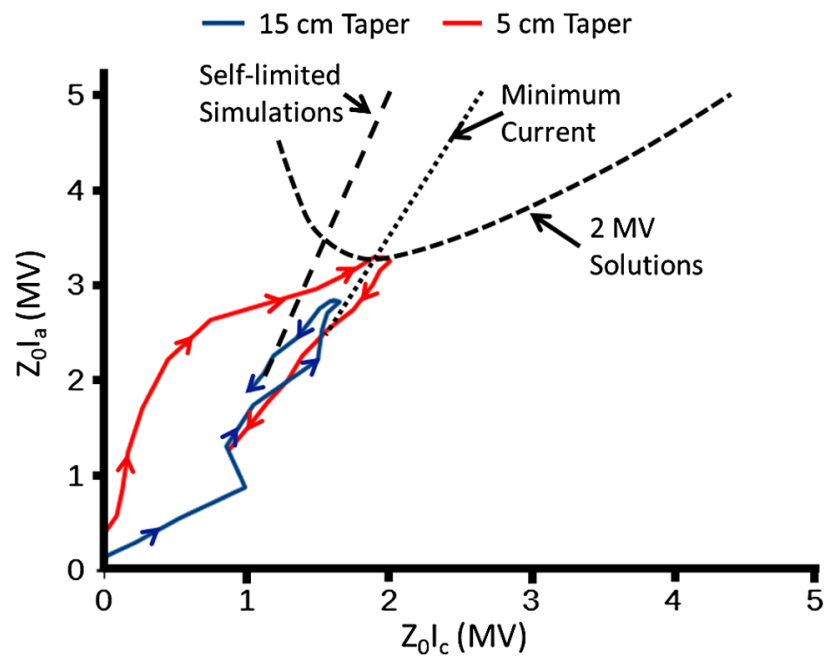

FIG. 6. Plot of $Z_{o} I_{a}$ versus $Z_{o} I_{c}$ as a function of time for the 5 -cm (inefficient) and $15-\mathrm{cm}$ (efficient) injector tapers in the MITE experiment, and the locus of $2 \mathrm{MV}$ solutions, the value of the minimum current, and the self-limited current from simulations by Ottinger and Schumer [21]. The arrows show the path from earliest to latest. achieve experimentally. Their simulations show that self-magnetic insulation begins from the overinsulated solution (the branch with the higher value of $I_{\text {cathode }}$ ) but the final equilibrium, represented by Eq. (3), resides on the saturated-flow branch (the branch with the lower value of $\left.I_{\text {cathode }}\right)$.

In contrast, the time-varying voltage on MITE precluded any equilibrium and MITE does not operate on the saturated-flow branch predicted by the Ottinger and Schumer simulations. As shown in Fig. 6, the MITE anode current $I_{a}$, as a function of the cathode current $I_{c}$, varied with time between the minimum current $I_{\min }$ and the "selflimited" current $I_{\mathrm{SL}}$ computed by Ottinger and Schumer. In addition, the self-magnetic insulation began on either the overinsulated branch or the underinsulated branchdepending on the geometry of the injector.

These results depend on an assumption that arises from the positons of the monitors. The MITE cathode and anode currents were measured 20 and $30 \mathrm{~cm}$, respectively, into the MITL. The measurements were $5 \mathrm{~cm}$ (five gap lengths) and $15 \mathrm{~cm}$ (15 gap lengths), respectively, downstream from the end of the injector, for both the 5-cm- and 15-cm-long injectors. Computer simulations indicated that five gap lengths were sufficient for the flow to reach a reasonably steady configuration, so we assume that the cathode current is the same at the position of the anode current measurement, $10 \mathrm{~cm}$ further downstream. The 6-m-long MITL was terminated in an electron beam diode operated at the $4.8-\Omega$ self-limited impedance of the MITL. There were no observable reflections from the diode load, so the experiment did investigate self-limited flow.

The more efficient injector approached peak voltage and power along the $I_{\min }$ curve and operated nearer to $I_{\min }$ than to $I_{\mathrm{SL}}$ at peak power. After peak power, the MITL operating point moved from $I_{\min }$ to $I_{\mathrm{SL}}$. The inefficient injector effectively launched an electron beam into the MITL with a minimal cathode current, so it approached peak power from the underinsulated branch, but reached peak power at $I_{\min }$ and then operated on the overinsulated branch before returning to $I_{\min }$ as the voltage decreased. Since $I_{\min }$ is at the intersection of the over- and underinsulated branches, we could have used either branch for the simulations of the long, self-limited MITL. Because we wish to use the same code to simulate load-dominated MITLs (which operate on the overinsulated branch), we used the overinsulated branch of Eq. (3) for our simulations. Ottinger and Schumer's equilibrium value of the self-limited current $I_{\mathrm{SL}}$ is very close to the minimum current $I_{\min }$, so the calculated performance is not strongly dependent on this choice.

Since Ottinger and Schumer's simulations were run until the flow reached equilibrium, Eq. (3) represents an equilibrium solution. The authors have produced a more generalized, time-dependent set of relationships [23], but those solutions also depend on the results of particle-in-cell simulations and have yet to be validated for different 
injector geometries. Therefore, we have developed a semiempirical circuit code to compare the electron losses observed in the MITE data with the results of circuit-code simulations of collisionless [Eq. (3)] and collisional [Eq. (4)] electron flow and with and without gap closure. We realize this is not a first-principles solution and offer it as an interim capability for the design of MITLs and for identifying what is probably important for efficient magnetic insulation.

With the efficient injector, the cathode current and anode current were almost equal until the electric field reached $\sim 1 \mathrm{MV} / \mathrm{cm}$, which occurred at $\sim 8 \mathrm{~ns}$ after electron emission usually starts at about $225 \mathrm{kV} / \mathrm{cm}$. We have seen delays in establishing full emission in other situations but have not investigated this potentially useful phenomenon further.

For the Screamer simulations, Eqs. (3) and (4) are assumed to be locally applicable in both space and time. In addition, the gap $d$ is assumed to close with a constant velocity $u=0$ or $2.5 \mathrm{~cm} / \mu \mathrm{s}$, and the effect of gap closure is assumed to be adequately incorporated by varying the vacuum wave impedance $Z_{o}(t)=Z_{o}[1-(u t / d)]$ [24] for both the collisionless and collisional theories. The cathode current $I_{\text {cathode }}$ is computed for each element in the MITL at each time step from the local voltage $V$, local anode current $I_{\text {anode }}$, and local vacuum wave impedance $Z_{o}=Z_{o}[1-(u t / d)]$ using Eq. (3) or (4). The electron current is calculated as $I_{e}=I_{\text {anode }}-I_{\text {cathode }}$. An increase in the vacuum wave impedance along the direction of electron flow decreases the supportable electron current. The decremented electron current represents the locally deposited electron loss plus retrapped electrons, but a circuit code cannot explicitly separate these two components.

The MITL solutions presented in Fig. 6 assume electron flow is already established. They do not describe the situation before electron emission begins or the situation (such as described by Fig. 7) in the loss front before insulation is established. Thus, both a loss front model and a model to describe flow losses during MITL operation after electron insulation are needed to model electron loss mechanisms in a circuit code.

Screamer [25] is a circuit code with lumped inductors, capacitors, and resistors. MITLs are simulated as series inductors separated by a capacitor and resistor to ground. The capacitors and inductors have fixed values; the resistor values are computed from the local current and voltage to simulate the losses associated with magnetic insulation. Screamer is often used to simulate MITLs in three different ways. They are, in order of increasing sophistication, (i) the BMITL (basic MITL) model (also called the racetrack model or MITL model in other papers), (ii) the $Z_{\text {Flow }}$ model, and (iii) the $Z_{\text {loss }}$ model.

The BMITL model simulates magnetic insulation by gradually reducing the space-charge-limited electron flow as the self-magnetic field at the anode exceeds $B_{\text {crit }}$ :
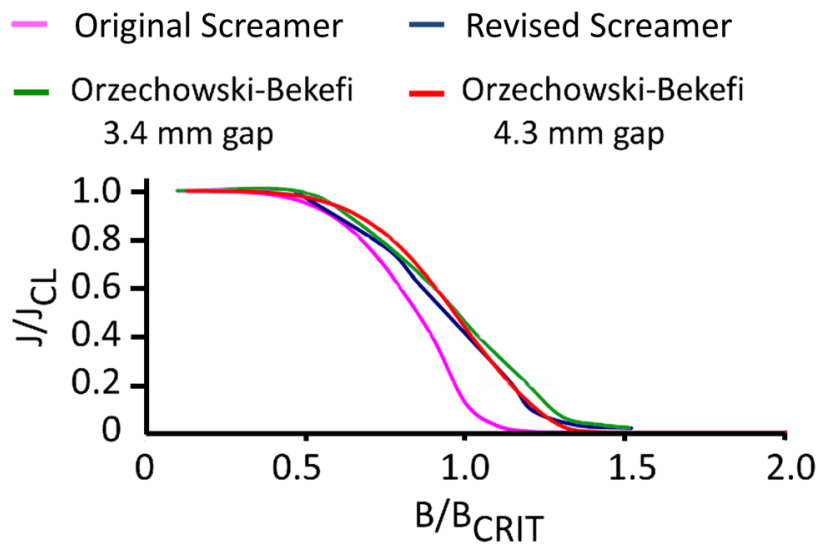

FIG. 7. The electron current density crossing the magnetic field (normalized to Child-Langmuir 1D space-charge-limited flow without a magnetic field) is shown as a function of the magnetic field at the anode $B_{\text {anode }}$ (normalized by the critical magnetic field for single particle magnetic insulation $B_{\text {crit }}$ ). Data from Orzechowski and Bekefi at about $300 \mathrm{KV}$ across two different anodecathode gaps are shown with the algorithm in the currently released version (original Screamer) and a slight modification used in this paper (revised Screamer).

$$
B_{\text {crit }}=\frac{m_{o} c}{e w d} \sqrt{\left(\frac{e V}{m_{o} c^{2}}\right)^{2}+\frac{2 e V}{m_{o} c^{2}}},
$$

in which $e$ and $m_{o}$ are the charge and rest mass of the electron, respectively, and $c$ is the speed of light in vacuum.

The BMITL model simulates the losses at the beginning of a current pulse in a long MITL with constant vacuum wave impedance. The model has no information about the electron flow behind the front, so it does not simulate any $Z_{\text {Flow }}$ losses predicted by Eq. (3) for collisionless flow or Eq. (4) for collisional flow.

The $Z_{\text {Flow }}$ model uses lossless tapered transmission lines (called TRLs in other papers) and a single resistor after the TRL [11]. The resistor is chosen to implement the often used assumption that "all the electron flow is lost before the load." For a $Z_{\text {Flow }}$ given by Eq. (1), the resistor has a value

$$
R=Z_{\text {Flow }} \frac{\sqrt{I_{\text {anode }}+I_{\text {cathode }}}}{\sqrt{I_{\text {anode }}-I_{\text {cathode }}}} .
$$

For example, MITE is modeled with $Z_{\text {Flow }}=6.0 \Omega$, vacuum wave impedance $Z_{o}=7.6 \Omega$, self-limited and matched impedance $Z_{\mathrm{SL}}=4.8 \Omega$, and $R=11.2 \Omega$ for the $Z_{\text {Flow }}$ model.

The $Z_{\text {loss }}$ model has multiple BMITL elements, instead of lossless TRLs, and uses a postprocessor to dump only the change in the supportable electron current [consistent with Eq. (3) or (4)] from the beginning to the end of each BMITL element. This model allows for distributed losses and allows for some of the electron flow, in principle, to be retrapped and reach the load. 
Each BMITL element is characterized by the vacuum wave impedance, the anode-cathode gap, the circumference, the electrical length tau, and the time resolution tresline. Both the BMITL model and the $Z_{\text {loss }}$ model assume that the anode-cathode gap is much smaller than the circumference and approximate the electric and magnetic fields as the average fields $(E=V / d$ and $B=\mu_{o} I_{\text {anode }} / w$, for the voltage $V$ across the gap $d$ of the MITL with circumference $w, \mu_{o}=4 \pi \times 10^{-7} \mathrm{H} / \mathrm{m}$, and the total current $=I_{\text {anode }}$ in the gap at that location.

Screamer divides each BMITL element into $N=$ tau/tresline nodes, computes the critical magnetic field $B_{\text {crit }}$ (at which an electron would be insulated in the absence of collective effects), and computes conductance as a function of the geometry and voltage $V$ at that node. The original Screamer (version 2.2) had an error that treated each BMITL element as a single line $(N=1)$, forcing users to explicitly specify many BMITLs to model a single MITL. The revised Screamer used for the calculations reported herein has corrected this error.

The critical current $I_{\text {crit }}=w B_{\text {crit } /} \mu_{o}$ and critical impedance $Z_{\text {crit }}=V / I_{\text {crit }}$ correspond to $B_{\text {crit }}$. Dividing $Z_{\text {crit }}$ by the vacuum wave impedance $Z_{o}$ makes the result independent of geometry but strongly dependent on the voltage [26]. The conductance of each resistor in the BMITL model smoothly decreases from Child-Langmuir flow as the magnetic field increases. One-dimensional theories by Lovelace and Ott [27] and by Creedon [18] predict the electron loss goes to zero when the magnetic field $B$ approaches and exceeds $B_{\text {crit }}$, as shown in Fig. 7.

In the original Screamer, the ratio of electron loss current density $J$ to $1 \mathrm{D}$ Child-Langmuir current density $J_{\mathrm{CL}}$ was derived from 3D particle-in-cell simulations and is also shown in Fig. 7.

Orzechowski and Bekefi [28] found that the electron flow is only $60 \%$ insulated at $B_{\text {crit }}$ and found microwave emission consistent with an instability that broadens the electron canonical momentum distribution. Electrons in the tail of the distribution cross the insulating magnetic field, but the fraction of electrons that reach the anode diminishes as the strength of the magnetic field increases. To improve the agreement with experimental data, we modified the algorithm for conductance as a function of $B / B_{\text {crit }}$ in Screamer to more closely agree with the results of Orzechowski and Bekefi. The resulting model is called the revised Screamer model, and the resulting normalized cross-field current density is shown in Fig. 7.

\section{A. Current transport efficiency through the MITE tapered convolute}

The transport efficiency of the MITE tapered convolute lets us test the assumption $[7,11,24,29]$ that the electron flow does not reach the load. The difference between the curve labeled MITE data and the curve labeled $Z_{\text {Flow }}$ model in Fig. 3 is a measure of the substantial retrapping of electron flow and contradicts the assumption of no retrapping. In the matched load case with $Z_{\mathrm{Load}} / Z_{\mathrm{o}_{-} \min }=0.63$, $\sim 60 \%$ of the electron flow was retrapped by this convolute, in which the vacuum wave impedance increased to a factor of 2.6 times the upstream $Z_{\mathrm{O}_{-} \min }$ over an axial distance of 30 gap spacings. Two-dimensional computer simulations by Hughes et al. [29] of the original (prerefurbishment) MITLs on $\mathrm{Z}$ also showed substantial retrapping of the electron flow in the upstream portion of the MITL, in which the vacuum wave impedance was slowly increasing with distance in the direction of the load. However, their simulations showed that the electron flow detaches from the cathode when the vacuum wave impedance increases too quickly - a factor of 2.0 times the upstream $Z_{\mathrm{o}_{-} \min }$ over a radial distance of ten gap spacings. They associated the retrapping with the diocotron instability [30]. With a gradually increasing impedance profile, the electrons stay close to the cathode, and the small oscillating electric field from the diocotron instability lets the electrons return to the cathode. With rapidly increasing impedance, the electron sheath separates from the cathode, and the even larger oscillations of diocotron instability are inadequate to return them to the cathode. The 3D simulations of the PHC in Ref. [12] confirm and further inform the problems with rapidly increasing impedance as a function of distance in magnetically insulated structures.

These results indicate that the electron flow in a section of the MITL with a very rapidly changing impedancesuch as the final, radial disk feed to a central load-will indeed be lost to the anode. Therefore, the design should minimize the electron flow going into any section of rapidly varying impedance-including the final disk feed.

The BMITL, $Z_{\text {Flow }}$, and the postprocessed $Z_{\text {Loss }}$ models are often used to calculate the current transport efficiency through a MITL. The data from the MITE tapered convolute experiment are used to examine the validity of the various models. The results are shown in Fig. 3. The MITE MITL vacuum wave impedance $Z_{o}=7.6 \Omega$. Its self-limited impedance is $4.8 \Omega$, which is also the impedance of the matched (highest power efficiency) load $Z_{\text {Load }}$ at the end of the convolute. The measured efficiency with a matched load (at $Z_{\text {Load }} / Z_{o}=0.63$ in Fig. 3) was only $85 \% \pm 7 \%$. However, designers often make the conservative assumption $[7,11,24,29]$ of the $Z_{\text {Flow }}$ model that all the electron flow at the end of the MITL is lost before the load; that assumption corresponds to a $52 \%$ efficiency at $Z_{\text {Load }} / Z_{o}=0.63$, as shown by the purple circle in Fig. 3. This assumption may be appropriate for the posthole convolute with its abrupt variation in geometry but is too conservative, as shown in Fig. 3, when the convolute is smoothly varying, which is the case in the MITE experiment.

The efficiency for all the experiments with the load impedance less than or equal to the matched impedance was $82 \% \pm 7 \%$, which shows that overly insulating the electron flow does not produce $100 \%$ efficient current flow. 
The minimum vacuum wave impedance $Z_{\mathrm{o}_{-} \min }=7.6 \Omega$ in the MITE experiment and is the impedance that determines the fraction of current in electron flow. The calculated load current (input current to the tapered convolute minus the calculated electron loss current in the postprocessed $Z_{\text {Loss }}$ model) is shown in Fig. 3 as " $Z_{\text {Loss }}$ " and gives $77 \%$ transport efficiency for a matched load, which is in reasonable agreement with the data shown in Fig. 3. The calculation correctly shows the transport efficiency decreasing with increasing $Z_{\text {Load }} /$ $\mathrm{Z}_{\mathrm{O}_{-} \text {min }}$ with no adjustable parameters but gives a higher than measured efficiency for $Z_{\text {Load }} / Z_{\mathrm{o}_{-} \min }>1$ and a $100 \%$ efficiency (versus $82 \% \pm 7 \%$ in the experiment) for $Z_{\text {Load }} / Z_{\mathrm{O}_{-} \min }<0.5$.

The $Z_{\text {Flow }}$ model in Screamer uses a single resistor $R$ to ground to estimate the $Z_{\text {Flow }}$ losses associated with the converging geometry. A value of $R=5.2 \Omega=0.68 Z_{\mathrm{o}_{-} \min }$ gives $I_{\text {Load }}=I_{\text {cathode }}$, as assumed in the model, but severely underestimates the efficiency for almost all the loads.

The collisional flow model was intended as a "worst case," and the Screamer results show that to be true. It gives the worst overall agreement with the MITE data.

None of the models give excellent agreement with the MITE data. The postprocessed $Z_{\text {Loss }}$ model is the most accurate and is useful for calculating the approximate distribution of the loss current density and the corresponding anode heating and ion emission. Therefore, the distribution of current density lost to the walls of the tapered convolute was modeled with the Screamer MITL model-postprocessed $Z_{\text {Loss }}$ model with the understanding that an accuracy of only $\sim 10 \%$ can be expected for $0.4<Z_{\text {Load }} / Z_{\mathrm{o}_{-} \min }<1.2$.

\section{B. Distribution of electron losses in MITE tapered convolute}

The MITE tapered convolute was modeled as a series of 153 MITLs, each of which has an electrical length of 10 ps. The load was $4.8 \Omega$, which matches the self-limited MITL impedance. The voltage and anode current for each element in the tapered convolute was computed with the Screamer MITL subroutine with and without gap closure and with both collisionless and collisional electron flow models. The results were processed with the postprocessed $Z_{\text {Loss }}$ model. The loss current density, averaged over the $35 \mathrm{~ns}$ pulse duration, was computed by using Eq. (3) (collisionless) or (4) (collisional) for the locations of the TLDs shown in Fig. 1(b). The results are compared to the experimental data in Fig. 8.

The original and revised (Orzechowski-Bekefi) Screamer MITL models with collisionless electrons and without gap closure are in reasonable agreement with the MITE data. The revision to improve the agreement with the Orzechowski-Bekefi data has a very minor effect for the MITE tapered convolute experiment.
- MITE Data

Standard Screamer without gap closure

- Orzechowski-Bekefi without gap closure

Orzechowski-Bekefi with gap closure

Collisional electron flow without gap closure

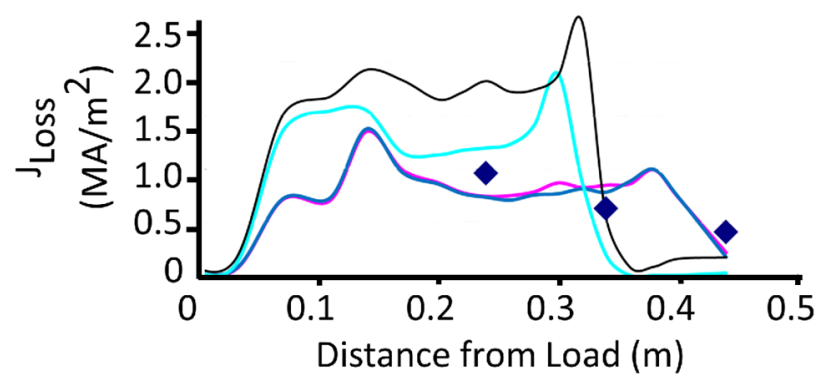

FIG. 8. Comparison of the computed time-integrated current density and the values measured on the sides of the MITE tapered convolute for four variations of the Screamer MITL model with $Z_{\text {Loss }}$ postprocessing.

The simulation with gap closure has the loss current density much less than the measured value at the $25-\mathrm{cm}$ position. Consequently, gap closure does not appear to be important in the MITE tapered convolute experiment, in agreement with the data from other experiments that are discussed in Sec. IV. The simulation with collisional electrons is indeed a worst case, as Styga et al. [21] intended, and does not agree with the MITE data. However, the disagreement for both of these models is solely dependent on the measurements at one position.

The maximum temperature rise $\Delta T$ in the anode of the tapered convolute is computed with Screamer by using two different models for the electron loss: (i) postprocessing using Eq. (3) and assuming collisionless electrons and (ii) equating the electron loss between any two distances from the load to the difference between the total currents $\left(\Delta I_{\text {Total }}\right)$ calculated by Screamer at those two distances. The results are shown in Fig. 9.

The postprocessed $Z_{\text {Loss }}$ calculation, which agrees with the MITE data in Figs. 3 and 8, is many times the premagnetic insulation losses from the MITL model alone, as shown in Fig. 9. Therefore, using the MITL model without the postprocessor dramatically underrepresents the losses.

The agreement between the computed values and the values measured on the sides of the MITE convolute in Fig. 8 suggests that Screamer adequately simulates the losses in a gradually tapering convolute when the geometry is smoothly varying. The disagreement with the larger values measured at the top of the convolute, as shown in Fig. 4, implies that Screamer cannot simulate the more complex electron flow in regions with a significant gradient in magnetic field perpendicular to the electron flowincluding PHCs. 
- With Postprocessing based on Eq. (3)

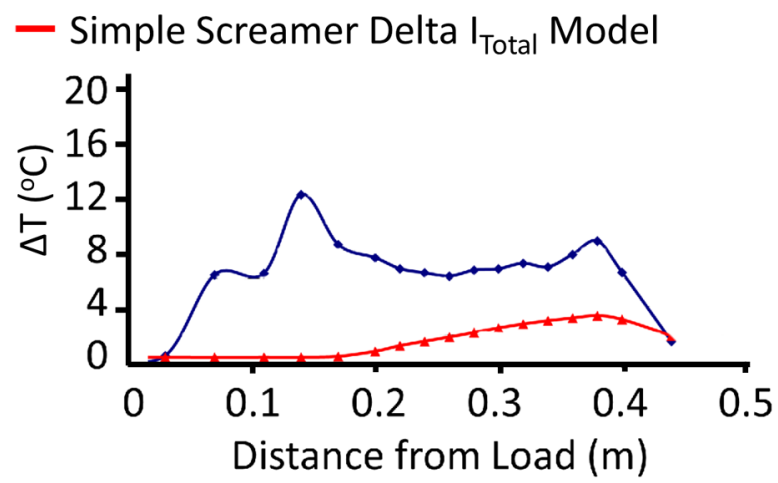

FIG. 9. Computed temperature rise in the anode of the MITE tapered convolute from the Screamer MITL model with the postprocessed $Z_{\text {Loss }}$ calculation based on Eq. (3) (blue) and without postprocessing (red).

The general agreement between MITE data from the tapered convolute and Screamer MITL simulations with the $Z_{\text {Loss }}$ postprocessing algorithm of Eq. (3) shows that the Screamer MITL model with the postprocessor for collisionless electron flow is a useful tool for the initial design of short MITLs which have an electrical length that is much less than the pulse length and have a smoothly varying impedance profile.

The results of decades of research and development on MITLs and the results of the MITE experiment let us offer a set of MITL requirements in the next section. We realize that some of the criteria will differ from common practice and hope that the differences will stimulate research to improve this version of the requirements.

\section{MITL REQUIREMENTS}

Stygar et al. [24,31] designed the four-level MITL with a PHC for the original $Z$ Machine. It proved to be an extremely reliable device that permitted precision experiments to be routinely performed on $Z$ for a decade. The following MITL design requirements for MITLs were developed from the criteria of that successful design and augmented by the results from other experimental and theoretical work as noted below.

1. Use bare stainless steel electrodes and keep the electric field $E<330 \mathrm{kV} / \mathrm{cm}$ for (a) the metal rings in the vacuum insulator stack and (b) the MITL cathode surfaces facing the vacuum insulator [24,31]. Implementing this requirement avoids electron emission that would alter the electric field grading in the vacuum stack and cause flashover or bulk breakdown of the vacuum insulator.

2. Design the MITL impedance profile to achieve a workable compromise between two conflicting constraints: (i) the MITL inductance must be low to provide efficient coupling between the generator and the load, but (ii) the electron current $I_{e}=I_{\text {anode }}-I_{\text {cathode }}$ must be small to minimize electron losses, anode plasma production, and ion losses. The first goal requires the MITL to have low vacuum wave impedance with small spacing between the electrodes, and the second goal requires large vacuum wave impedance with large gaps. The balance between these two competing requirements is achieved [24,31] when

(i) $I_{e}<10 \%$ of $I_{\text {anode }}$ at $I_{\text {anode }}=67 \%$ of $I_{\text {peak }}$;

(ii) $I_{e}<7 \%$ of $I_{\text {anode }}$ at peak current; and

(iii) $I_{e}<10 \%$ of $I_{\text {anode }}$ at $t=5 \mathrm{~ns}$ before peak current.

3. Keep the anode-cathode gap in the power feed that is being bombarded by an intense flux of x-rays large enough to meet the experimental requirements for $\mathrm{x}$-ray energy and power without shorting. The required gap scales with the peak current. For a 30 MA peak current, Stygar et al. [32] recommend the minimum gap should be $4.7 \mathrm{~mm}$ for maximum x-ray energy and $2.7 \mathrm{~mm}$ for maximum x-ray power. These minimum gaps are unnecessarily restrictive for portions of the MITL that are not being bombarded by high fluxes of $\mathrm{x}$-rays or particles and that have a magnetic field that is much larger than $0.5 \mathrm{~T}$. The restrictive gap requirements are based on a $2.5 \mathrm{~cm} / \mu \mathrm{s}$ cathode closure velocity with the magnetic field freely diffusing through the cathode plasma [24,31]. Low-power laboratory experiments [33-37] with 0-0.5 $\mathrm{T}$ magnetic fields in some or all of the anode-cathode gap do show an average closure velocity of $\sim 2.5 \mathrm{~cm} / \mu$ s or higher.

However, experiments have shown that the expansion velocity is not uniform and it decreases with increasing magnetic field. Orzechowski and Bekefi [28] found that the plasma expansion velocity was initially zero for $20 \mathrm{~ns}$ and then became $6 \mathrm{~cm} / \mu$ s without an applied magnetic field. An applied magnetic field of only $1 \mathrm{~T}$ was clearly sufficient to stop plasma motion across the gap in a uniform field MITL, as inferred from the change of conductance with time, for 40-ns pulses and 2.3-4.3 mm anode-cathode gaps.

In addition, their magnetohydrodynamic simulations of hydrogen plasma on the cathode, with and without an applied magnetic field, show complex plasma motion as a shock is formed in the plasma and the temperature increases with time. For example, the expansion is reversed after the plasma has penetrated a maximum of $\sim 1 \mathrm{~mm}$ into the anode-cathode gap with a $1 \mathrm{~T}$ magnetic field.

In the extreme case of a point-plane gap, with its enhanced current density, plasma heating, plasma temperature, and pressure at the cathode tip, an insulating magnetic field of $2.8 \mathrm{~T}$ reduces the cathode plasma expansion velocity [38].

Bliss et al. [39] examined gap closure with magnetic fields $\sim 400 \mathrm{~T}$. They used laser shadowgraphy on $Z$ to study cathode plasma motion across a $4 \mathrm{~mm}$ anode-cathode MITL gap with a peak current of 19 MA. The average closure velocity was $<0.22 \mathrm{~cm} / \mu$ s while the current was increasing before the main $\mathrm{x}$-ray pulse. When the current decreased and the associated magnetic field compressing the magnetized cathode plasma decreased, the compressed 
magnetic field embedded in the plasma drove closure at $\sim 6 \mathrm{~cm} / \mu \mathrm{s}$. The expansion of the compressed plasma after the current maximum was reached has been studied theoretically by Spielman, Chantrenne, and McDaniel [40]. Stinnett et al. [37] observed a similar effect at only $0.5 \mathrm{~T}$ magnetic fields, in which the closure velocity reached $20 \mathrm{~cm} / \mu \mathrm{s}$ as the current decreased.

Therefore, the local magnetic field $>0.5 \mathrm{~T}$ inhibits gap closure for spatially smooth geometries while the current is increasing. The $0.5 \mathrm{~T}$ threshold for suppressing gap closure is appropriate for the few eV plasma typical of MITLs without a large energy flux to the electrodes. The threshold must be larger if an $\mathrm{x}$-ray pulse heats the cathode and anode plasmas to more than $\sim 4 \mathrm{eV}$.

Stygar et al. [32] show that even a $1 \mathrm{~mm}$, strongly magnetically insulated gap bombarded by a high-flux of x-rays remains open - as conservatively defined by no decrease in the impedance of the gap-until $~ 93$ ns into the current pulse, which is $<2 \mathrm{~ns}$ before peak $\mathrm{x}$-ray power. In these experiments, the electrode plasmas are heated to 90-100 eV by the $\mathrm{x}$-ray pulse, but the intense magnetic field was sufficient to keep the anode-cathode gap open until the current decreased. However, they inferred that the magnetic inhibition of gap closure was possibly less effective for 19 MA than it was for 13 MA total current.

The overall geometry, including the minimum anodecathode gap as a function of position, is also constrained by the net motion of the electron sheath under $\operatorname{gradB}$ drift (which forces electrons across the gap) and $\mathbf{E} \times \mathbf{B}$ drift (which moves the electrons towards the load). Tapering the cathode and anode to keep the net guiding center motion away from the anode has improved the current to the load on Proto II [41].

In summary, the minimum gap as a function of radius is constrained by all of these effects: (i) increased electron flow and associated losses with decreasing gap, (ii) thermal expansion of the cathode plasma, (iii) heating of the cathode plasma by local x-ray sources, (iv) reduction of closure velocity with increasing magnetic field, (v) increase of closure velocity with decreasing current, (vi) magnetic field diffusion into the plasma, and (vii) $\operatorname{grad} \mathbf{B}$ and $\mathbf{E} \times \mathbf{B}$ drifts. Each application must be examined to determine the optimum gap based on calculations of all these effects. Otherwise, use a closure velocity of $2.5 \mathrm{~cm} / \mu \mathrm{s}$ if the magnetic field $B<0.5 \mathrm{~T}$ and use a closure velocity of $0 \mathrm{~cm} / \mu$ s for $B \gg 0.5 \mathrm{~T}$ and $d\left(B^{2}\right) / d t>0$ (i.e., increasing current). However, if $B \gg 0.5 \mathrm{~T}$ and $d\left(B^{2}\right) / d t<0$, design for a closure velocity of $20 \mathrm{~cm} / \mu \mathrm{s}$.

4. To the extent possible, avoid strong gradients in the vacuum wave impedance versus distance along the Poynting vector. As discussed in Sec. III A, electron retrapping is most effective in gradually tapering MITL structures. This requirement is incompatible with the PHC [10-12].

5. For each level of MITL leading into a PHC, make the vacuum wave impedance $Z_{o}=0.1 * V_{\max } / I_{\text {_at_Vmax }}$ for the peak voltage $V_{\max }$ and the current in that particular MITL at peak voltage $I_{\text {_at_Vmax }}$ to minimize the electron flow into the PHC [24,31]. For Sandia's Z accelerator, this requirement gives a $1 \mathrm{~cm}$ gap at $10 \mathrm{~cm}$ radius.

6. Use 5-mm radial gaps in the cylindrical return current conductor surrounding an imploding plasma load [24,31]. This requirement is consistent with requirement 3 for the typical 100-150 ns implosions of wire arrays. For shorter and longer pulse drivers and different loads, the requirement can be adjusted in proportion to the pulse duration and the many factors discussed in requirement 3.

7. Design for the highest inductance load planned for the facility and expect to see new loss mechanisms when extending operations to higher power or higher energy experiments. This conservatism increases the range of experiments that the facility can support and provides margin for mitigating the effects of new discoveries.

Attempts to increase power to a high-performance load by approximately $50 \%$ on $Z$ in 2005 were foiled by a new loss mechanism that is still being researched in 2015. To illustrate the effects of the loss and motivate the discussion of the requirements for self-magnetically insulated power flow discussed in this paper, consider an imploding-plasma load with a very stressing combination of high initial inductance and large time rate of change of inductance. It is a $1-\mathrm{cm}$-long $z$ pinch with an initial radius of $3 \mathrm{~mm}$ and a mass of $200 \mathrm{mg}$. The initial inductance inside the $8 \mathrm{~cm}$ radius (the radius of the PHC in the standard four-level MITL on $\mathrm{Z}$ in 2005) is $5 \mathrm{nH}$. The computed change of inductance is $4.6 \mathrm{nH}$ at a convergence ratio of 10 . Analysis by Jennings [11] of experiments on $Z$ at similar power levels indicates $\mathrm{a} \ll 1-\Omega$ resistive shunt develops at the PHC at about 192 ns into the current pulse, when the voltage begins to spike to $>2.5 \mathrm{MV}$ at the PHC. The calculated performance of the four-level MITL and PHC on Sandia's $Z$ accelerator with this extreme surrogate load and loss mechanism is shown in Figs. 10 and 11.

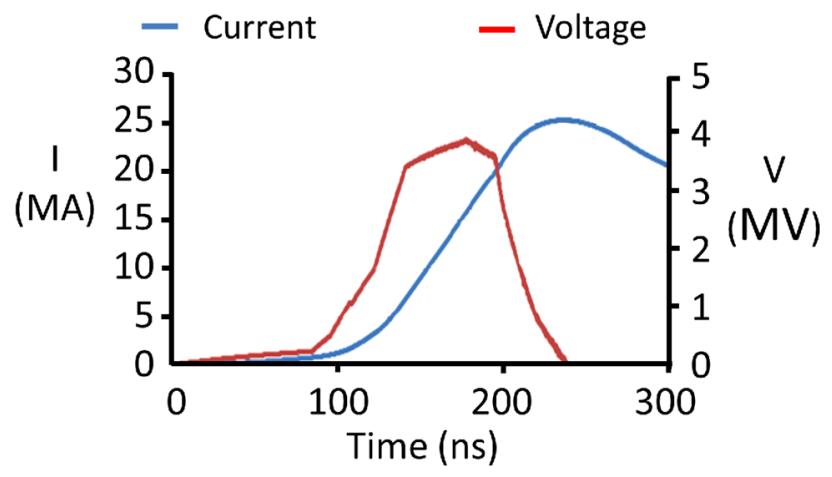

FIG. 10. The voltage and current at the MITL input versus time are shown as computed with Screamer for the surrogate load on the $Z$ Machine with the standard four-level MITL and PHC. The voltage source was inferred from shot 1774 . 


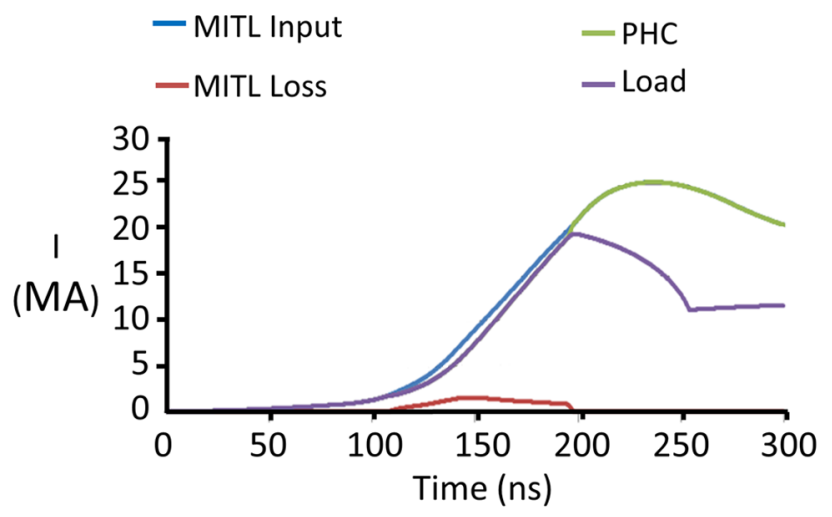

FIG. 11. The effect of the $Z_{\text {Flow }}$ loss resistance immediately after the PHC and beginning at 190 ns for the very stressing surrogate load is illustrated with Jennings's model of the current loss. The losses are shown as the difference between the PHC current and the load current. The losses in the MITL before the PHC are shown as MITL loss, which goes to almost zero in the simulation after the $Z_{\text {Flow }}$ current loss begins.

This very stressing load will be used to illustrate some of the requirements below. Although this loss was ultimately found to be inconsistent with simple space-charge-limited ion losses, ion losses must be considered and are discussed in requirement 9 .

8. Ensure that the spatial resolution in circuit simulations used to design the MITL is sufficient for the pre-magneticinsulation loss front to be distributed over more than one element. Since the loss current typically extends over about 3 times the gap spacing [15], resolving the mesh in the direction of the Poynting vector to be better than the local anode-cathode gap satisfies this requirement and provides a reasonable estimate of the local electron heating of the anode in circuit simulations.

9. To mitigate ion current losses [42-49], compute the anode heating from the combination of resistive heating from the anode current and electron and negative ion loss to the anode and ensure that the temperature is $<400^{\circ} \mathrm{C}$ $[36,45,46,51-53]$ over as much of the MITL anode as practical. After the heating has been calculated, assume that a space-charge-limited source of protons is located on the anode wherever the temperature exceeds $400{ }^{\circ} \mathrm{C}$ and compute the electron and ion currents self-consistently with a particle-in-cell simulation. Exclude regions in which protons are magnetically insulated. Compute the remaining ion losses and the effect of the ion space charge on the electron flow.

The fraction of the anode surface that emits ions is not necessarily large, and the losses may be source limited. The problem has to be considered as a system, with simulations of a specific design for each application. Schumer, Ottinger, and Olson [54] have published such a study for the challenging case of $z$-pinch fusion. They conclude that the ion losses are manageable for a 60 MA driver with a 2 mm gap.
The resistive heating of the anode at small radius (i.e., at large current per unit width) is a major issue in designs for $z$-pinch fusion [54]. The total current heats the anode over the entire pulse. Long pulses, high currents per unit width, and the resistivity of the stainless steel or aluminum anodes can cause resistive heating to dominate in some cases.

The temperature increase $\Delta T$ from resistive heating can be estimated as

$$
\Delta T=\frac{\mu_{o} \pi}{\rho \tau c_{p}} \int_{0}^{t}\left(\frac{I(t)}{w}\right)^{2} d t,
$$

in Standard International units and in which $\mu_{\mathrm{o}}=4 \pi \times 10^{-7} \mathrm{H} / \mathrm{m}, \rho$ is the mass density of the anode, $\tau$ is the time to peak current, $c_{p}$ is the specific heat of the metal electrode, and $I(t) / w$ is the current per unit width as a function of time. The integral is taken from the start of the current pulse at time $t=0$ to time $t$. For example, an aluminum anode reaches $400{ }^{\circ} \mathrm{C}$ in $100 \mathrm{~ns}$ if the linearly rising current per unit width is $0.54 \mathrm{MA} / \mathrm{cm}$ (or $34 \mathrm{MA}$ flowing radially at $10 \mathrm{~cm}$ radius) at time $100 \mathrm{~ns}$. The resulting temperature is accurate to $\sim 30 \%$ and is a conservatively high estimate for most pulse shapes of interest.

The electron heating should be calculated with a particlein-cell code or a circuit code, e.g., the Screamer code with the $Z_{\text {Loss }}$ postprocessing described in this paper. Negative ion heating is less mature and is discussed in requirement 18 .

After the heating has been calculated, assume that a space-charge-limited source of protons is located on the anode wherever the temperature exceeds $400^{\circ} \mathrm{C}$ and compute the electron and ion currents self-consistently in particle-in-cell simulations. Hughes et al. [29] and Madrid et al. [12] found that the ion space charge enhances the magnetically insulated electron current by a factor of $2-3$. If circuit simulations are used for preliminary calculations, assume the resulting ion current is enhanced (by magnetically insulated electrons) a factor of 2 over monopolar space-charge-limited ion flow and assume the electron sheath current in this region is enhanced (by the ion space charge) a factor of 3 times the value computed from the postprocessed $Z_{\text {Loss }}$ model described in Sec. III.

Exclude regions in which protons are magnetically insulated. Self-magnetically insulated power flow with ions, but without electrons, has been considered by Ottinger and Schumer [48]. Wang et al. [55] consider both ion and electron flow and show that the electron flow is negligible when the ions are magnetically insulated-even though the ion flow enhances the electron flow by a factor of about 2. Their equations reduce to those of Ottinger and Schumer when the small electron current is neglected.

For almost all cases of interest, the minimum total current $I_{\min }$ and the self-limited current $I_{\mathrm{SL}}$ calculated by 
Ottinger and Schumer for insulated ion flow are nearly the same. Therefore, Eqs. (9)-(11) in Ref. [48] are useful in determining when the ion flow becomes magnetically insulated, after which the ion losses are inhibited. For example, the shot illustrating PHC losses in Ref. [11] has a voltage of 2.2 MV and a current of $15 \mathrm{MA}$ at the PHC when the losses begin. The corresponding value of $I_{\min }$ for insulating protons in a $10-\mathrm{cm}$ radius disk feed with a 1-cm gap is $10.7 \mathrm{MA}$, so the protons are already well insulated in the disk feed when the losses begin. The insulated ion current is only $25 \mathrm{kA}$, so the corresponding total insulated ion and electron current would be only $75 \mathrm{kA}$ [55]. This finding is consistent with the conclusion in Ref. [12] that the MITLs on $Z$ must be emitting ions to a radius of approximately $20 \mathrm{~cm}$ to explain the losses. That portion of the MITL should not have been heated to $400{ }^{\circ} \mathrm{C}$ and should not be emitting ions if the electron flow is stable. However, unstable electron flow from the injector could conceivably be causing an anode plasma before the $\mathrm{PHC}$, as discussed in requirement 11.

Finally, anode plasma provides the possibility of anode plasma expansion by ion charge exchange with neutrals, as described by Prono et al. [56]. Their measurements of the impedance collapse in a macrosecond-duration ion diode with reflexing electrons is consistent with a gap closure velocity of $10 \mathrm{~cm} / \mu \mathrm{s}$ or more, and their particle detectors record neutrals with energies of $10-25 \mathrm{keV}$ reaching the cathode. Filuk et al. [57] spectroscopically measured energetic lithium neutrals near the anode in an applied- $B$ ion diode with a 50-ns duration ion beam. Gomez [58] used spectral absorption to measure the distribution of neutral hydrogen in the PHC of the $Z$ accelerator and found the neutral number density decreases with distance from the cathode, in contrast to the results of Filuk et al. Therefore, the role of energetic neutrals in MITL and PHC losses has not been established. Of course, eliminating the anode plasma mitigates the formation and effects of energetic neutrals from the anode. If that is not possible, then energetic neutrals require further study.

10 . For a very conservative design, assume $100 \%$ of the computed electron current $I_{e}=I_{\text {anode }}-I_{\text {cathode }}$ at the end of the MITL is lost to the anode [7,24,29]. This assumption is appropriate for a PHC with a large variation in the vacuum wave impedance, as discussed in Sec. III A. For a more gradual transition, much of the electron current can be retrapped. For the example shown in Fig. 3 (in which the vacuum wave impedance $Z_{o}$ increases to 2.6 times its value $Z_{\mathrm{o}_{-} \min }$ at the beginning of the convolute over a distance of approximately 30 gap separations), only $\sim 40 \%$ of that electron current is lost in the convolute. Making the most conservative assumption may delay discovery of a new loss mechanism or delay the resources needed to understand and mitigate such losses.

The calculations of $I_{e}$ in requirements 8 and 9 are best done with a fully electromagnetic, 2D or 3D particle-in-cell simulation. Useful, preliminary calculations can be done with a circuit simulation using the $Z_{\text {Loss }}$ model described in Sec. III with the multiplicative factors for the ion and electron current described in requirement 9.

11. Taper the electron injector region at the beginning of the MITL so that the electron emission originates over at least ten Larmor radii to maximize $Z_{\text {Flow }}$ and minimize the electron loss [15]. The losses addressed by requirement 11 arise from a kinetic instability [59] of the electron flow and may contribute to the losses mitigated by requirements 9 and 10. For a load-dominated MITL-PHC combination, the electron losses caused by a high-gradient injector may cause ion emission in the MITL before the PHC and account for the PHC losses, as postulated by Madrid et al. [12].

Although this requirement was originally formulated for long, self-limited MITLs, many load-limited MITLs operate with the input at, or above, the self-limited value, as shown in Fig. 12 for $Z$ with a realistic but stressing load, and are also susceptible to theses kinetic losses. Modern, load-limited MITLs, including the Z MITL as shown in Fig. 5(b), do not meet this requirement, because simulations of their MITLs have not shown this kinetic loss. Since these losses have not been observed in our state-of-the-art particle-in-cell simulations of the MITE geometry but were observed in the MITE experiments, the fact that they have not been observed in simulations of other geometries is not justification for ignoring this requirement.

Orzechowski and Bekefi [28] investigated magnetically insulated electron flow in a coax insulated with an applied magnetic field as a test of the MITL models by Lovelace and Ott [27] and Creedon [18]. They found that the loss current density was accompanied by microwaves generated by an electron instability, which provided the canonical momentum transfer that lets electrons cross the insulating magnetic field. Although they investigated azimuthal

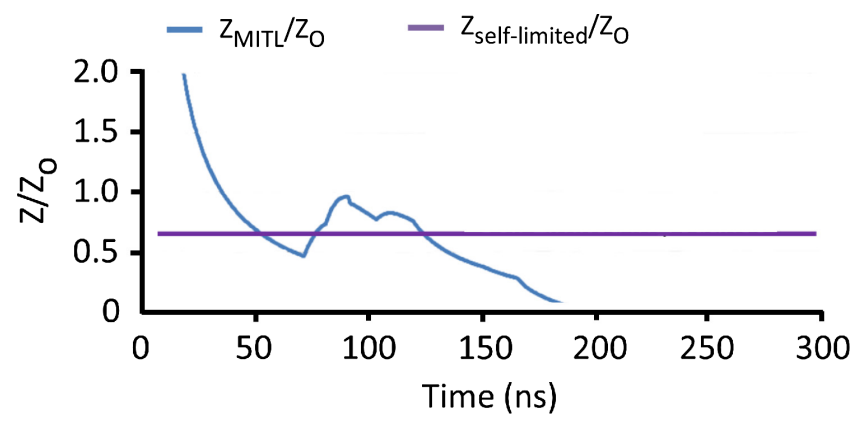

FIG. 12. The operational $Z / Z_{o}(Z$ is the voltage divided by the current at the input to the MITLs and $Z_{o}=0.69 \Omega$ is the equivalent minimum vacuum wave impedance of the four-level MITLs in parallel) for the surrogate load on $Z$ with the standard four-level MITL and PHC. The value for self-limited flow at $2 \mathrm{MV}$ is shown for comparison. With this load, the outer portions of the $Z$ MITLs operate near the self-limited value for the first third of its power pulse. 
electron flow with an axial magnetic field and a radial electric field, the local physics is very similar to the MITL problem of axial electron flow with an azimuthal magnetic field and a radial electric field. Electrons flow for many betatron wavelengths in both configurations. However, in contrast to the case of axial electron flow, there was no variation in the gap spacing with distance along the direction of electron flow. Orzechowski and Bekefi found that the equivalent of the self-limited impedance for efficient magnetic insulation was $75 \%$ of the value calculated from a simple $B_{\text {crit }}$ model.

Shope et al. [26] experimented with self-limited flow in a MITL whose electrical length was much less than the pulse duration. They found that the self-limited flow impedance was $35 \%-75 \%$ of the value calculated from $B_{\text {crit }}$. The entrance to the MITL was very abrupt, so the axial extent of the first-generation electron emission was comparable to the gap spacing; the energy transport was inefficient, in contrast to the results by Orzechowski and Bekefi with the equivalent of electron injection in a uniform electric field.

Long self-limited MITL experiments on MITE [15] provided data on five geometries; two were efficient and three were inefficient, as presented in Sec. II C. In the most abrupt and least efficient design, bands of damage on the cathode and electron losses to the anode between 53 and 121 gap separations into the MITL were consistent with an electron instability causing the loss. When the entrance to the MITL was modified to a more gradual taper (increasing the length over which electrons were injected), the transport was efficient for a distance of at least 600 times the anodecathode gap. In general, the more gradual injectors (in which the electron flow was launched over a larger number of Larmor radii) were more efficient.

Equation (3) was used to process the MITE injector data [15] and the four injectors of the standard four-level MITL on $Z[24,31]$ to find the profile of injected electron current (normalized by the total electron current) as a function of the distance into the MITL (normalized by the Larmor radius). Representative results are shown in Fig. 5(b). The profile with inefficient transport (at $Z_{\text {Flow }}=0.6 Z_{\mathrm{o}_{-} \min }$ ) on MITE is shown in red, and the profile for efficient transport (at $Z_{\text {Flow }}=0.71 Z_{\mathrm{o}_{-} \min }$ ) is shown in blue. The safe region is to the right of the blue line, and the inefficient region is to the left of the red line in Fig. 5(b). A comparison of the MITE and $Z$ injector profiles implies that the $Z$ MITLs are susceptible to the kinetic electron losses.

The results of the MITE injector experiments are shown in Fig. 13 as the operating points on plots of the fraction of current flowing in electrons $\left(I_{e} / I_{\text {anode }}\right)$ versus $Z_{\mathrm{Flow}} / Z_{\mathrm{O}_{-} \min }$ from Eq. (2), and $Z=0.63 Z_{\mathrm{o}_{\text {_min }}}$ from Shope et al. [26] for $V=2 \mathrm{MV}$ and also observed in Ref. [15].

Our simulations of the $Z$ injectors show that the electron flow that is lost at the PHC is consistent with the empirical value [11] of $Z_{\text {Flow }}=0.25 \Omega=0.363 Z_{\mathrm{o}_{-} \min }$. Since the more gradual tapers in the electron injector on MITE had

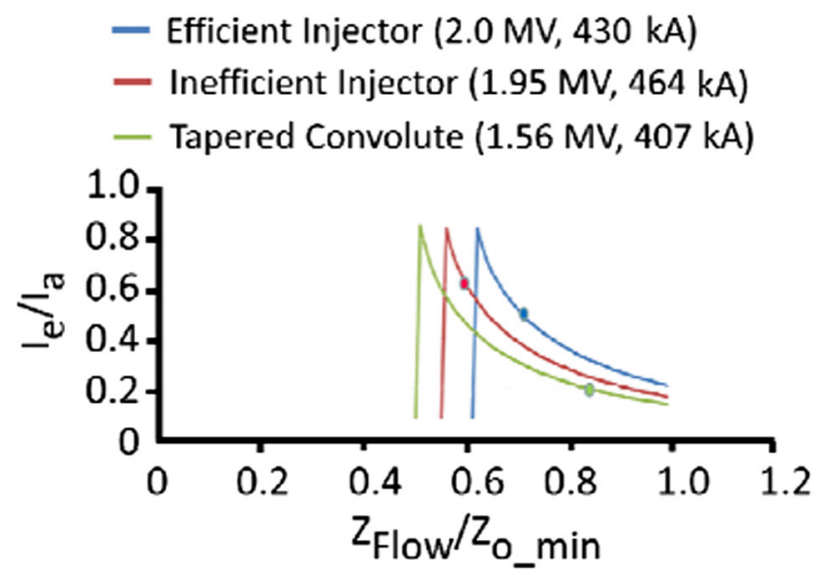

FIG. 13. The fraction of current flowing in electrons is shown as a function of $Z_{\mathrm{Flow}} / Z_{\mathrm{O}_{-} \text {min }}$ for the two MITE injector experiments and the tapered convolute discussed in Sec. II B. The operating points are shown by the circles on the respective curves. All have $Z_{\mathrm{o}_{-} \min }=7.6 \Omega$.

higher values of $Z_{\mathrm{Flow}} / Z_{\mathrm{O}_{-} \text {min }}$ and higher transport efficiencies, redesign of the $Z$ MITL to include a gradual injector, in accord with the safe profile shown in Fig. 5(b), may increase $Z_{\text {Flow }}$ to as much as $0.84 Z_{\mathrm{O}_{-} \text {min }}$ and reduce the electron losses accordingly, even though doing so for an accelerator like $Z$ adds about $1 \mathrm{nH}(8 \%)$ to the inductance.

In summary, applying requirement 11 to both selflimited and load-dominated MITLs mitigates the risk associated with the injector. Currently, load-dominated MITLs do not satisfy this requirement, and some suffer from losses with modestly high impedance loads. Until the dominant cause of the losses is understood, we adopt requirement 11 in the interest of making a robust design.

12. Make the cathode of the MITL out of stainless steel [24,31], because electron emission from stainless steel is much more uniform and reproducible than emission from bare aluminum (with its insulating oxide coating), alodined aluminum (with its conductive chromate conversion coating that deteriorates with multiple shots), or fragile coatings like graphite.

13. Use the results in Stygar et al. [60] to check on the energy lost to the electrodes by thermal heating, magnetic field diffusion, and electrode motion. If the experimental parameters are outside of the domain studied in Ref. [60], i.e., current/width $>10 \mathrm{MA} / \mathrm{cm}$ or pulse durations $>300 \mathrm{~ns}$, then use a validated magnetohydrodynamic code to check on the energy lost. Although the losses are less than $10^{5} \mathrm{~J}$ on $Z$ in the domain studied by Stygar et al. [60], and the load energy is typically $>10^{6} \mathrm{~J}$, extrapolation of their formulas well beyond the domain of demonstrated validity gives much larger losses. Therefore, such cases must be studied in detail to predict the load energy.

14. Ensure the electric field is below the threshold for emission everywhere that the magnetic field is insufficient to insulate the electrons $[24,31]$. One of the unsuccessful 
injectors in the MITE experiment had azimuthally nonuniform magnetic field density, and electron losses were observed in the low magnetic field regions. The losses were eliminated by moving the nonuniform region of magnetic field to a region in which the electric field was below the threshold for electron emission.

Spielman et al. [61] found a similar loss in a convolute for Proto II. The orientation of the electric field transitioned from vertical in the vacuum insulator stack to horizontal in the main MITL to vertical in the disk feed to the load. The magnetic field profile from the currents flowing through the junctions (between the horizontal electrodes of the vacuum insulator section and the vertical electrodes of the MITL) left large regions uninsulated. Spielman et al. observed large electron losses in those uninsulated regions. Increasing the vacuum gap between the cathode and the anode in these regions mitigated the losses but produced lossy magnetic nulls near the convolute combining these separate MITLs.

Instead of cutting away the metal on the cathode, requirement 14 keeps the electric field in these uninsulated regions below the emission threshold, as was successfully demonstrated in the MITE experiment. Although this requirement increases the inductance somewhat, it avoids potential losses associated with the magnetic nulls.

15. If the MITL section closest to the experiment will be damaged on each shot (as they are in multimegajoule experiments), make the center section of the MITL low cost and expendable. Refurbishment is expensive and time consuming and presents a risk to the experimenter, whose hardware is designed for an undeformed contact surface. Although this requirement is not possible for PHCs, modern casting techniques make disposable center sections both practical and cost advantageous for alternative MITL geometries, like the clamshell MITL [62].

16. Current contacts between sections of the MITL and the experimental hardware should have deformable metal gaskets with sufficient pressure to ensure arcing does not initiate additional losses or have contacts well removed from the highly stressed anode-cathode gap.

17. The hardware that experiences current per unit width in excess of $0.5 \mathrm{MA} / \mathrm{cm}$ must be electropolished, vacuum baked, and gold coated to provide highly reliable power flow to the experiment [63]. Since it is desirable to minimize this expensive treatment, the MITL terminates in a standard flange, at a radius of $8 \mathrm{~cm}$ on $Z$, to accept the specially treated hardware from the experimenter.

18. Negative ion emission from the cathode must not be allowed to (i) turn on too much of the anode and cause excessive ion-current loss and anode plasma closure, (ii) enhance cathode plasma closure by charge exchange transport of neutrals, or (iii) transport electrons into the anode-cathode gap that can be stripped from the negative ion by photons, electrons, or ion collisions and accumulate in the gap. In the third case, the accumulated electron space charge shields the cathode and enhances the electric field near the anode-potentially causing greatly enhanced positive ion current, whose charge sustains the negative ion current.

Continuous negative ion current densities of $10^{4} \mathrm{~A} / \mathrm{m}^{2}$ have been developed [64,65] with low-work-function metal cathodes. The cathode plasma in a MITL provides a zero-work-function equivalent, so it is not too surprising that source-limited negative ions have been observed in carbon-coated MITLs with current densities of up to $3 \times 10^{5} \mathrm{~A} / \mathrm{m}^{2}$ and proportional to the cube of current per unit $I / w \sim 1 \mathrm{MA} / \mathrm{m}[66,67]$. If that scaling holds to the $10-100 \mathrm{MA} / \mathrm{m}$ in PHC and diode feeds, then the cathode may supply space-charge-limited currents of negative ions that can produce anode plasma.

The following negative-ion controlled process may, or may not, contribute to the excessive current losses observed in posthole convolutes on $Z$ at Sandia National Laboratories [10-12]:

(a) The cathode plasma is formed when the electric field on the cathode exceeds $\sim 300 \mathrm{kV} / \mathrm{cm}$ and forms a zero-work-function source of negative ions.

(b) Negative ion current density $\left(J^{-}\right)$from the cathode plasma increases with the cube of the current per unit width [67] until they are space-charge limited.

(c) After $\sim 400 \mathrm{~J} / \mathrm{g}$ is deposited into the anode ( $\sim 30 \mathrm{~ns}$ into the $Z$ current pulse at the PHC), the anode plasma is formed and positive, space-charge-limited ion current flows.

(d) Negative ions transport attached electrons across magnetic field lines.

(e) The negative ion number density $\left(J^{-} / u\right)$ is greatest near the cathode, where their velocity $u$ is least.

(f) Photon, electron, and ion collisions with the negative ions during transport detach electrons, which accumulate predominately near the cathode-distorting the potential distribution in the gap and increasing the positive ion current density required to give $E=0$ at the anode.

(g) The increased positive ion flow partially neutralizes the electron space charge near the cathode, maintaining the negative ion current.

(h) The accumulating electron charge in the gap causes the total (predominately positive) ion current to become important at about $65 \mathrm{~ns}$ into the $Z$ pulse at PHC in our preliminary estimate of the loss current possible with this process.

Although this process might cause current losses in PHCs, and excessive current losses in a PHC-like geometry have been attributed to negative ions [68] without actually measuring them in that experiment, negative ions have not yet been studied with adequate diagnostics in PHCs. Gomez's measurements [58] (of the decrease in hydrogen-neutral density with increasing distance from the cathode in the $Z$ PHC) are consistent with the negative ion hypothesis. However, Gomez's results are also 
consistent with cathode-plasma motion as a contributor to the PHC losses [10]. Without more study, the negative ion requirement for efficient MITLs remains somewhat speculative in the list of conservative requirements.

Not all these requirements are equally necessary, or even possible, in all MITL applications. For example, the standard four-level MITL and PHC on Z does not meet requirements 4,7 , and 11 . However, each of the 18 should be examined in the design of a MITL and in the investigation of excessive losses.

\section{CONCLUSIONS}

We have compared the results of the MITE experiment with modern theoretical treatments of magnetic insulation. We found significant discrepancies between the data and the interpretations of computer simulations of modern devices. Simulations have not yet reproduced the effects caused by the injector geometry and by other structures, such as posthole convolutes, that rapidly vary the vacuum wave impedance with distance along the Poynting vector. In general, slowly varying structures are more efficient than rapidly varying structures. Published assumptions that the electron flow is lost before the load and that the gap closure velocity is $\sim 2.5 \mathrm{~cm} / \mu \mathrm{s}$ are not supported by the data from MITE and other experiments. Such assumptions make identification and mitigation of new losses more difficult. Neither analytic nor computational theories have yet considered the potential effects of negative ions' transporting electrons across insulating magnetic fields and enhancing positive ion current losses in posthole convolutes. We have suggested a set of 18 requirements for magnetically insulated power flow and encourage others to improve them based on their own experimental, computational, or analytical results.

\section{ACKNOWLEDGMENTS}

Sandia National Laboratories is a multiprogram laboratory operated by Sandia Corporation, a wholly owned subsidiary of Lockheed Martin Company, for the U.S. Department of Energy's National Nuclear Security Administration under Contract No. DE-AC04-94AL85000. We gratefully acknowledge both reviewers for their useful critical reviews, which led to a much better contribution.

[1] D. B. Sinars, R. W. Lemke, M. E. Cuneo, S. V. Lebedev, E. M. Waisman, W. A. Stygar, B. Jones, M. C. Jones, E. P. Yu, J. L. Porter, and D. F. Wenger, Phys. Rev. Lett. 100, 145002 (2008).

[2] J. P. Davis, J. Appl. Phys. 99, 103512 (2006).

[3] M. D. Knudson, M. P. Desjarlais, and D. H. Dolan, Science 322, 1822 (2008).

[4] C. L. Ruiz, G. W. Cooper, S. A. Slutz, J. E. Bailey, G. A. Chandler, T. J. Nash, T. A. Mehlhorn, R. J. Leeper,
D. Fehl, A. J. Nelson, J. Franklin, and L. Ziegler, Phys. Rev. Lett. 93, 015001 (2004).

[5] D. R. Alexander, IEEE Trans. Nucl. Sci. 50, 565 (2003).

[6] T. D. Pointon, W. A. Stygar, R. B. Spielman, H. C. Ives, and K. W. Struve, Phys. Plasmas 8, 4534 (2001).

[7] C. W. Mendel, Jr., T. D. Pointon, M. E. Savage, D. B. Seidel, I. Magne, and R. Vezinet, Phys. Plasmas 13, 043105 (2006).

[8] T. D. Pointon, W. L. Langston, and M.E. Savage, in Proceedings of the 16th IEEE Pulsed Power and Plasma Science Conference, Albuquerque, 2007, edited by E. Schamiloglu and F. Peterkin (IEEE, Piscataway, NJ, 2007), p. 165.

[9] W. A. Stygar, M. E. Cuneo, D. I. Headley, H. C. Ives, R. J. Leeper, M. G. Mazarakis, C. L. Olson, J. L. Porter, and T. C. Wagoner, Phys. Rev. ST Accel. Beams 10, 030401 (2007).

[10] D. V. Rose, D. R. Welch, T. P. Hughes, R. E. Clark, and W. A. Stygar, Phys. Rev. ST Accel. Beams 11, 060401 (2008).

[11] C. A. Jennings, J. P. Chittenden, M. E. Cuneo, W. A. Stygar, D. J. Ampleford, E. M. Waisman, M. Jones, M. E. Savage, K. R. LeChien, and T. C. Wagoner, IEEE Trans. Plasma Sci. 38, 529 (2010).

[12] E. A. Madrid, D. V. Rose, D. R. Welch, R. E. Clark, C. B. Mostrom, W. A. Stygar, M. E. Cuneo, M. R. Gomez, T. P. Hughes, T. D. Pointon, and D. B. Seidel, Phys. Rev. ST Accel. Beams 16, 120401 (2013).

[13] A. A. Kim, M. G. Mazarakis, V. A. Sinebryukhov, B. M. Kovalchuk, V. A. Visir, S. N. Volkov, F. Bayol, A. N. Bastrikov, V. G. Durakov, S. V. Frolov, V. M. Alexeenko, D. H. McDaniel, W. E. Fowler, K. LeChien, C. Olson, W. A. Stygar, K. W. Struve, J. Porter, and R. M. Gilgenbach, Phys. Rev. ST Accel. Beams 12, 050402 (2009).

[14] C. L. Olson, in Landolt-Boernstein Handbook on Energy Technologies, edited by W. Martienssen (Springer-Verlag, Berlin, 2005), Vol. III/3; in Fusion Technologies, edited by (Physics Institute, University of Bonn, Germany, 2005), Chap. 9, pp. 493-526.

[15] J. P. VanDevender, J. Appl. Phys. 50, 3928 (1979).

[16] J. P. VanDevender, J. T. Crow, B. G. Epstein, D. H. McDaniel, C. W. Mendel, Jr., E. L. Neau, J. W. Poukey, J. P. Quintenz, D. B. Seidel, and R. W. Stinnett, Physica B +C (Amsterdam) 104, 167 (1981).

[17] P. L. Dreike, E. J. T. Burns, S. A. Slutz, J. T. Crow, D. J. Johnson, P. R. Johnson, R. J. Leeper, P. A. Miller, L P. Mix, D. B. Seidel, and D. F. Wenger, J. Appl. Phys. 60, 878 (1986).

[18] J. M. Creedon, J. Appl. Phys. 48, 1070 (1977).

[19] C. W. Mendel, Jr., J. Appl. Phys. 50, 3830 (1979).

[20] C. W. Mendel, Jr., D. B. Seidel, and S. A. Slutz, Phys. Fluids 26, 3628 (1983); R. I. Lawconnel and J. Nen, Phys. Fluids B 2,629 (1990); C. W. Mendel, Jr., M. E. Savage, D. M. Zagar, W. W. Simpson, T. W. Grasser, and J.P. Quintenz, J. Appl. Phys. 71, 3731 (1992).

[21] P. F. Ottinger and J. W. Schumer, Phys. Plasmas 13, 063109 (2006).

[22] W. A. Stygar, T. C. Wagoner, H. C. Ives, P. A. Corcoran, M. E. Cuneo, J. W. Douglas, T. L. Gilliland, M. G. Mazarakis, J. J. Ramirez, J. F. Seamen, D. B. Seidel, and 
R. B. Spielman, Phys. Rev. ST Accel. Beams 9, 090401 (2006).

[23] P. F. Ottinger and J. W. Schumer, IEEE Trans. Plasma Sci. 35, 154 (2007).

[24] W. A. Stygar, R. B. Spielman, G. O. Allshouse, C. Deeney, D. R. Humphreys, H. C. Ives, F. W. Long, T. H. Martin, M. K. Matzen, D. H. McDaniel, C. W. Mendel, Jr., L. P. Mix, T. J. Nash, J. W. Poukey, J. J. Ramirez, T. W. L. Sanford, J. F. Seamen, D. B. Seidel, J. W. Smith, D. M. Van De Valde, R. W. Wavrik, P. A. Corcoran, J. W. Douglas, I. D. Smith, M. A. Mostrom, K. W. Struve, T. P. Hughes, R. E. Clark, R. W. Shoup, T. C. Wagoner, T. L. Gilliland, and B. P. Peyton, in Proceedings of the 11th IEEE International Pulsed Power Conference, Baltimore, 1997, edited by G. Cooperstein and I. Vitkovitsky (IEEE, Piscataway, NJ, 1997), p. 591.

[25] M. L. Kiefer, K. L. Shaw, K. W. Struve, and M. M. Widner, Screamer, A Pulsed Power Design Tool, User's Guide, Version 2.2, May 1999, Sandia National Laboratories, Albuquerque, NM (unpublished).

[26] S. Shope, J. W. Poukey, K. D. Bergeron, D. H. McDaniel, A. J. Toepfer, and J. P. VanDevender, J. Appl. Phys. 49, 3675 (1978).

[27] R. V. Lovelace and E. Ott, Phys. Fluids 17, 1263 (1974).

[28] T. J. Orzechowski and G. Bekefi, Phys. Fluids 19, 43 (1976).

[29] T. P. Hughes, R. E. Clark, B. V. Oliver, T. D. Pointon, and W. A. Stygar, in Proceedings of the 14th IEEE International Pulsed Power Conference, 2003, Vol. 1, edited by M. Gieesselmann and A. Neuber (IEEE, Piscataway, NJ, 2003), pp. 622-625.

[30] J. D. Lawson, The Physics of Charged Particle Beams (Clarendon, Oxford, 1977), p. 410.

[31] W. A. Stygar, P. A. Corcoran, H. C. Ives, R. B. Spielman, J. W. Douglas, B. A. Whitney, M. A. Mostrom, T. C. Wagoner, C. S. Speas, T. L. Gilliland, G. A. Allshouse, R. E. Clark, G. L. Donovan, T. P. Hughes, D. R. Humphreys, D. M. Jaramillo, M. F. Johnson, J. W. Kellogg, R. J. Leeper, F. W. Long, T. H. Martin, T. D. Mulville, M. D. Pelock, B. P. Peyton, J. W. Poukey, J. J. Ramirez, P. G. Reynolds, J. F. Seamen, D. B. Seidel, A. P. Seth, A. W. Sharpe, R. W. Shoup, J. W. Smith, D. M. Van De Valde, and R. W. Wavrik, Phys. Rev. ST Accel. Beams 12, 120401 (2009).

[32] W. A. Stygar, H. C. Ives, D. L. Fehl, M. E. Cuneo, M. G. Mazarakis, J. E. Bailey, G. R. Bennett, D. E. Bliss, G. A. Chandler, R. J. Leeper, M. K. Matzen, D. H. McDaniel, J. S. McGurn, J. L. McKenney, L. P. Mix, D. J. Muron, J. L. Porter, J. J. Ramirez, L. E. Ruggles, J. F. Seamen, W. W. Simpson, C. S. Speas, R. B. Spielman, K. W. Struve, J. A. Torres, R. A. Vesey, T. C. Wagoner, T. L. Gilliland, M. L. Horry, D. O. Jobe, S. E. Lazier, J. A. Mills, T. D. Mulville, J. H. Pyle, T. M. Romero, J. J. Seamen, and R. M. Smelser, Phys. Rev. E 69, 046403 (2004).

[33] R. B. Baksht, S. P. Vavilov, and M. N. Urbazaev, Izv. Vyssh. Uchebn. Zaved., Fiz. 2, 140 (1973) [Sov. Phys. J. 16, 266 (1973)]; see discussion in G. A. Mesyats and D. I. Proskurovsky, Pulsed Discharges in Vacuum, Vol. 5 of the Springer Series on Atoms and Plasmas (SpringerVerlag, Berlin, 1989), Sec. 4.4, pp. 72-74.
[34] D. D. Hinshelwood, IEEE Trans. Plasma Sci. 11, 188 (1983).

[35] Ya. Ya. Yurike, V. F. Puchkarev, and D. I. Proskurovskii, Sov. Phys. J. 16, 293 (1973).

[36] R. K. Parker, R. E. Anderson, and C. V. Duncan, J. Appl. Phys. 45, 2463 (1974).

[37] R. W. Stinnett, M. A. Palmer, R. B. Spielman, and R. Bengston, IEEE Trans. Plasma Sci. 11, 216 (1983).

[38] R. B. Baksht and G. G. Mesyats, Izv. Vyssh. Uchebn. Zaved., Fiz. 7, 144 (1970) [Sov. Phys. J. 13, 969 (1970)]; See discussion in G. A. Mesyats and D. I. Proskurovsky, Pulsed Discharges in Vacuum, Vol. 5 of the Springer Series on Atoms and Plasmas (SpringerVerlag, Berlin, 1989), Sec. 4.4, pp. 72-74.

[39] D. E. Bliss, M. E. Cuneo, B. M. Jones, K. W. Struve, W. A. Stygar, E. M. Waisman, and S. J. Chantrenne, in Proceedings of the 16th IEEE Pulsed Power and Plasma Science Conference, Albuquerque, 2007 (unpublished).

[40] R. B. Spielman, S. Chantrenne, and D. H. McDaniel, in Proceedings of the 16th IEEE Pulsed Power and Plasma Science Conference, Albuquerque, 2007, edited by E. Schamiloglu and F. Peterkin (IEEE, Piscataway, NJ, 2007), pp. 937-941.

[41] D. A McDaniel and M. Clauser, Sandia National Laboratories (private communication).

[42] G. M. McCracken, R. S. Barton, and W. Dillon, Nuovo Cimento Suppl. 5, 146 (1967).

[43] D. W. Swain, S. A. Goldstein, J. G. Kelly, and G. R. Hadley, J. Appl. Phys. 46, 4604 (1975).

[44] J. G. Kelly, S. A. Goldstein, and D. W. Swain, J. Appl. Phys. 46, 4726 (1975).

[45] A. E. Blaugrund, G. Cooperstein, and S. A. Goldstein, Phys. Fluids 20, 1185 (1977).

[46] T. W. L. Sanford, J. A. Halbleib, J. W. Poukey, A. L. Pregenzer, R. C. Pate, C. E. Heath, R. Mock, G. A. Mastin, D. C. Ghiglia, T. J. Roemer, P. W. Spence, and G. A. Proulx, J. Appl. Phys. 66, 10 (1989).

[47] R. A. Vesey, T. D. Pointon, M. E. Cuneo, T. A. Mehlhorn, J. E. Bailey, D. J. Johnson, and W. A. Stygar, Phys. Plasmas 6, 3369 (1999).

[48] P. F. Ottinger and J. W. Schumer, Phys. Plasmas 13, 063101 (2006).

[49] P. F. Ottinger and J. W. Schumer, IEEE Trans. Plasma Sci. 35, 154 (2007).

[50] P. Spence, K. Triebes, R. Genuario, and D. Pellinen, in Proceedings of the International Topical Conference on Electron Beam Research and Technology, edited by G. Yonas (Sandia National Laboratories, Albuquerque, NM, 1976) (Sandia National Laboratories Report No. SAND76-5122), p. 346.

[51] Q. D. Dakin and J. Benford, Physics International internal memo, San Leandro, CA, 1976 (unpublished).

[52] C. R. McClenahan, T. P. Wright, J.P. Quintenz, R. C. Backstrom, and P. W. Spence, Sandia National Laboratories Report No. SAND85-0740, 1985 (unpublished).

[53] M. E. Cuneo, R. M. Gilgenbach, and M. L. Brake, IEEE Trans. Plasma Sci. 15, 375 (1987).

[54] J. W. Schumer, P. F. Ottinger, and C. L. Olson, IEEE Trans. Plasma Sci. 34, 2652 (2006). 
[55] H. Wang, L. Meng, D. Liu, L. Liu, and C. Yang, Phys. Plasmas 19, 103506 (2012).

[56] D. S. Prono, H. Ishizuka, E. P. Lee, B. W. Stallard, and W. C. Turner, J. Appl. Phys. 52, 3004 (1981).

[57] A. B. Filuk, J. E. Bailey, A. L. Carson, D. J. Carson, P. Lake, T. A. Mehlhorn, L. P. Mix, T. J. Renk, W. A. Stygar, and Y. Maron, Phys. Rev. Lett. 77, 3557 (1996).

[58] M. R. Gomez, Ph.D. dissertation University of Michigan, http://gradworks.umi.com/34/58/3458853.html, 2011.

[59] K. D. Bergeron and J. W. Poukey, J. Appl. Phys. 50, 4996 (1979).

[60] W. A. Stygar, S. E. Rosenthal, H. C. Ives, T. C. Wagoner, G. O. Allshouse, K. E. Androlewicz, G. L. Donovan, D. L. Fehl, M. H. Frese, T. L. Gilliland, M. F. Johnson, J. A. Mills, D. B. Reisman, P. G. Reynolds, C. S. Speas, R. B. Spielman, K. W. Struve, A. Toor, and E. M. Waisman, Phys. Rev. ST Accel. Beams 11, 120401 (2008).

[61] R. B. Spielman, W. W. Hsing, D. H. McDaniel, P. W. Spence, and K.W. Nielson, in Proceedings of the 6th IEEE Pulsed Power Conference, Arlington, VA,
1987, edited by B. H. Bernstein and P. J. Turchi (IEEE, New York, 1987), IEEE Catalog No. 87CH2522-1, pp. 639-642.

[62] J. P. VanDevender, W. L. Langston, M. F. Pasik, R. S. Coats, T. D. Pointon, D. B. Seidel, C. A. Jennings, G. R. McKee, and L. X. Schneider, in Proceedings of the Pulsed Power Conference, Chicago, 2011, edited by B. V. Oliver (IEEE, New York, 2011), pp. 1003-1008.

[63] R. B. Spielman (private communication).

[64] Y. I. Bel'chenke, G. I. Dimov, and V. G. Dudnikov, Berkeley National Laboratory (BNL) Report No. 50727, p. 79.

[65] J. R. Hiskes and P. J. Schneider, BNL Report No. 51304, p. 15.

[66] J. P. VanDevender, R. W. Stinnett, and R. J. Anderson, Appl. Phys. Lett. 38, 229 (1981).

[67] R. W. Stinnett and T. Stanley, J. Appl. Phys. 53, 3819 (1982).

[68] W. U. Hanyu, Z. Zeng, L. Wang, and N. Guo, Plasma Sci. Technol. 16, 625 (2014). 